\title{
Discrete Modified Projection Method for Urysohn Integral Equations with Smooth Kernels
}

\author{
Rekha P. KULKARNI *and Gobinda RAKSHIT ${ }^{\dagger}$
}

\begin{abstract}
Approximate solutions of linear and nonlinear integral equations using methods related to an interpolatory projection involve many integrals which need to be evaluated using a numerical quadrature formula. In this paper, we consider discrete versions of the modified projection method and of the iterated modified projection method for solution of a Urysohn integral equation with a smooth kernel. For $r \geq 1$, a space of piecewise polynomials of degree $\leq r-1$ with respect to an uniform partition is chosen to be the approximating space and the projection is chosen to be the interpolatory projection at $r$ Gauss points. The orders of convergence which we obtain for these discrete versions indicate the choice of numerical quadrature which preserves the orders of convergence. Numerical results are given for a specific example.
\end{abstract}

Key Words : Urysohn integral operator, Interpolatory projection, Gauss points, Nyström Approximation

AMS subject classification : 45G10, 65J15, 65R20

*Department of Mathematics, I.I.T. Bombay, Powai, Mumbai 400076, India, rpk@math.iitb.ac.in,

${ }^{\dagger}$ gobindarakshit@math.iitb.ac.in 


\section{Introduction}

Let $X=L^{\infty}[a, b]$, and consider a Urysohn integral operator

$$
\mathscr{K}(x)(s)=\int_{a}^{b} \kappa(s, t, x(t)) d t, \quad s \in[a, b], x \in X,
$$

where $\kappa(s, t, u)$ is a continuous real valued function defined on

$$
\Omega=[a, b] \times[a, b] \times \mathbb{R} .
$$

Then $\mathscr{K}$ is a compact operator from $L^{\infty}[a, b]$ to $C[a, b]$. Assume that for $f \in C[a, b]$,

$$
x-\mathscr{K}(x)=f
$$

has a unique solution $\varphi$.

We are interested in approximate solutions of the above equation. We consider projection methods associated with a sequence of interpolatory projections converging to the Identity operator pointwise.

For $r \geq 1$, let $X_{n}$ denote the space of piecewise polynomials of degree $\leq r-1$ with respect

to a uniform partition of $[a, b]$ with $n$ subintervals. Let $h=\frac{b-a}{n}$ denote the length of each subinterval of the above partition. Let $Q_{n}: C[a, b] \rightarrow X_{n}$ denote the interpolation operator at $r$ Gauss points. Then in the collocation method, (1.2) is approximated by

$$
\varphi_{n}^{C}-Q_{n} \mathscr{K}\left(\phi_{n}^{C}\right)=Q_{n} f
$$

The iterated collocation solution is defined as

$$
\varphi_{n}^{S}=\mathscr{K}\left(\phi_{n}^{C}\right)+f
$$

In Grammont et al [1] the following modified projection method is investigated:

$$
\varphi_{n}^{M}-\mathscr{K}_{n}^{M}\left(\phi_{n}^{M}\right)=f
$$

where

$$
\mathscr{K}_{n}^{M}(x)=Q_{n} \mathscr{K}(x)+\mathscr{K}\left(Q_{n} x\right)-Q_{n} \mathscr{K}\left(Q_{n} x\right) .
$$


It is a generalization of the modified projection method in the linear case, which was proposed in Kulkarni [13]. The iterated monified projection solution is defined as

$$
\tilde{\varphi}_{n}^{M}=\mathscr{K}\left(\phi_{n}^{M}\right)+f
$$

If $\frac{\partial \kappa}{\partial u} \in C^{r}(\Omega)$ and $f \in C^{r}[a, b]$, then the following orders of convergence can be obtained from Atkinson-Potra [6]:

$$
\left\|\varphi-\varphi_{n}^{C}\right\|_{\infty}=O\left(h^{r}\right), \quad\left\|\varphi-\varphi_{n}^{S}\right\|_{\infty}=O\left(h^{2 r}\right)
$$

The following estimates are obtained in Grammont et al [11]: Let $f \in C^{2 r}[a, b]$.

If $\frac{\partial \kappa}{\partial u} \in C^{2 r}(\Omega)$ and then

$$
\left\|\varphi-\varphi_{n}^{M}\right\|_{\infty}=O\left(h^{3 r}\right)
$$

whereas if $\frac{\partial \kappa}{\partial u} \in C^{3 r}(\Omega)$, then

$$
\left\|\varphi-\tilde{\varphi}_{n}^{M}\right\|_{\infty}=O\left(h^{4 r}\right)
$$

In practice, it is necessary to replace the integral in the definition of $\mathscr{K}$ by a numerical quadrature formula, giving rise to a discrete version of the above methods. The discrete version of the iterated collocation method is considered in Atkinson-Flores [4]. Our aim is to investigate a choice of a numerical quadrature rule which preserves the above orders of convergence in the discrete versions of the modified projection and the iterated modified projection methods.

We choose a composite numerical quadrature formula with a degree of precision $d$ and with respect to a uniform partition of $[a, b]$ with $m$ subintervals. Let $\tilde{h}$ denote the length of each subinterval of this partition. The discrete modified projection solution and the iterated discrete modified projection solution are denoted repectively by $z_{n}^{M}$ and $\tilde{z}_{n}^{M}$. We prove the following orders of convergence. Let $\kappa \in C^{d}(\Omega)$, and $f \in C^{d}[a, b]$.

$$
\begin{aligned}
& \text { If } \frac{\partial^{2} \kappa}{\partial u^{2}} \in C^{2 r}(\Omega) \text {, then } \\
& \left\|\varphi-z_{n}^{M}\right\|_{\infty}=O\left(\max \left\{\tilde{h}^{d}, h^{3 r}\right\}\right),
\end{aligned}
$$

whereas if $\frac{\partial^{2} \kappa}{\partial u^{2}} \in C^{3 r}(\Omega)$, then

$$
\left\|\varphi-\tilde{z}_{n}^{M}\right\|_{\infty}=O\left(h^{r} \max \left\{\tilde{h}^{d}, h^{3 r}\right\}\right) .
$$


Thus, the orders of convergence in (1.9) and (1.10) are preserved provided the numerical quadrature rule is so chosen that $\tilde{h}^{d}=h^{3 r}$.

In Chen et al [7] discrete versions of the modified projection and the iterated modified projection methods for solutions of linear integral equations are considered. They consider

a slightly restrictive case where the same uniform partition of $[a, b]$ is considered to define a composite numerical quadrature formula and an interpolatory projection, that is $\tilde{h}=h$. We allow these partitions to be different. More precisely, we choose $m=n p, p \in \mathbb{N}$, instead of $m=n$.

The emphasis of this paper is on the solution of Urysohn integral equations and we include the results about the linear case for the sake of completeness and for their use to treat the nonlinear case.

The paper has been arranged in the following way. In Section 2.1, we describe the Nyström approximation of $\mathscr{K}$ obtained by replacing the integral by a numerical quadrature. In Section 2.2, this approximation is used to define the discrete versions of projection methods (1.3)-(1.7). Section 3 is devoted to approximate solutions of linear integral equations using the discrete versions of the modified projection and the iterated modified projection methods. The order of convergence for the discrete modified projection solution of a Urysohn integral equation is obtained in Section 4, whereas the discrete iterated modified projection method is investigated in Section 5. Numerical results are given in Section 6.

\section{Methods of Approximation}

\subsection{Nyström Approximation}

Assume that the kernel $\kappa$ of $\mathscr{K}$ defined by (1.1) is such that

$$
\frac{\partial^{2} \kappa}{\partial u^{2}} \in C(\Omega)
$$

It is also assumed that 1 is not an eigenvalue of the compact linear operator $\mathscr{K}^{\prime}(\varphi)$ so that $\left(I-\mathscr{K}^{\prime}(\varphi)\right)^{-1}: C[a, b] \rightarrow C[a, b]$ is a bounded linear operator. 
Let $m \in \mathbb{N}$, and consider the following uniform partition of $[a, b]$ :

$$
a=s_{0}<s_{1}<\cdots<s_{m}=b .
$$

Let

$$
\tilde{h}=s_{k}-s_{k-1}=\frac{b-a}{m}, k=1, \ldots, m \text {. }
$$

Consider a basic quadrature rule

$$
\int_{0}^{1} f(t) d t \approx \sum_{i=1}^{\rho} w_{i} f\left(\mu_{i}\right)
$$

which has a degree of precision $2 r-1$ or higher.

Let

$$
\zeta_{i}^{j}=s_{j-1}+\mu_{i} \tilde{h}, \quad i=1, \ldots, \rho, \quad j=1, \ldots, m .
$$

A composite integration rule with respect to the partition (2.1) is then defined as

$$
\int_{a}^{b} f(t) d t=\sum_{j=1}^{m} \int_{s_{j-1}}^{s_{j}} f(t) d t \approx \tilde{h} \sum_{j=1}^{m} \sum_{i=1}^{\rho} w_{i} f\left(\zeta_{i}^{j}\right) .
$$

The error in the numerical quadrature is assumed to be of the following form:

There is an integer $d \geq 2 r$ such that if $f \in C^{d}[a, b]$, then

$$
\left|\int_{a}^{b} f(t) d t-\tilde{h} \sum_{j=1}^{m} \sum_{i=1}^{\rho} w_{i} f\left(\zeta_{i}^{j}\right)\right| \leq C_{1}\left\|f^{(d)}\right\|_{\infty} \tilde{h}^{d},
$$

where $f^{(d)}$ denotes $d$ th derivative of $f$ and $C_{1}$ is a constant independent of $\tilde{h}$.

If the basic quadrature rule in (2.2) is chosen to be the Gaussian quadrature with $\rho=r$ or the Newton-Cotes quadrature with $\rho=2 r$, then (2.5) is satisfied with $d=2 r$.

We replace the integral in (1.1) by the numerical quadrature formula (2.4) and define the Nyström operator as

$$
\mathscr{K}_{m}(x)(s)=\tilde{h} \sum_{j=1}^{m} \sum_{i=1}^{\rho} w_{i} \kappa\left(s, \zeta_{i}^{j}, x\left(\zeta_{i}^{j}\right)\right) .
$$

Then $\mathscr{K}_{m}$ has the following properties.

1. $\mathscr{K}_{m}: C[a, b] \rightarrow C[a, b]$ are completely continuous operators.

2. $\mathscr{K}_{m}$ is a colloctively compact family: $\mathscr{B} \subset C([a, b])$ bounded implies that the set $\left\{\mathscr{K}_{m}(\mathscr{B}): m \geq 1\right\}$ is relatively compact in $C[a, b]$. 
3. At each $x \in C[a, b], \mathscr{K}_{m}(x) \rightarrow \mathscr{K}(x)$ as $m \rightarrow \infty$.

4. $\left\{\mathscr{K}_{m}\right\}$ is an equicontinuous family at each $x \in C[a, b]$.

5. For $m \geq 1, \mathscr{K}_{m}$ are twice Fréchet differentiable:

$$
\mathscr{K}_{m}^{\prime}(x) v(s)=\tilde{h} \sum_{j=1}^{m} \sum_{i=1}^{\rho} w_{i} \frac{\partial \kappa}{\partial u}\left(s, \zeta_{i}^{j}, x\left(\zeta_{i}^{j}\right)\right) v\left(\zeta_{i}^{j}\right), \quad s \in[a, b] .
$$

and

$$
\mathscr{K}_{m}^{\prime \prime}(x)\left(v_{1}, v_{2}\right)(s)=\tilde{h} \sum_{j=1}^{m} \sum_{i=1}^{\rho} w_{i} \frac{\partial^{2} \kappa}{\partial u^{2}}\left(s, \zeta_{i}^{j}, x\left(\zeta_{i}^{j}\right)\right) v_{1}\left(\zeta_{i}^{j}\right) v_{2}\left(\zeta_{i}^{j}\right), \quad s \in[a, b] .
$$

Let $\kappa \in C^{d}(\Omega)$ and $f \in C^{d}[a, b]$. Then $\mathscr{K}$ is a compact operator from $L^{\infty}[a, b]$ to $C^{d}[a, b]$ and the exact solution $\varphi$ of (1.2) belongs to $C^{d}[a, b]$. From (2.5) we conclude that

$$
\left\|\mathscr{K}(\varphi)-\mathscr{K}_{m}(\varphi)\right\|_{\infty}=O\left(\tilde{h}^{d}\right)
$$

In the Nyström method, (1.2) is approximated by

$$
x_{m}-\mathscr{K}_{m}\left(x_{m}\right)=f .
$$

We quote the following result from Atkinson [1].

Fix $\delta>0$ and define

$$
B(\varphi, \delta)=\left\{x:\|x-\varphi\|_{\infty} \leq \delta\right\}
$$

There exists a positive integer $m_{0}$ such that for $m \geq m_{0}$, the equation (2.10) has a unique solution $\varphi_{m}$ in $B(\varphi, \delta)$ and

$$
\left\|\varphi-\varphi_{m}\right\|_{\infty}=O\left(\tilde{h}^{d}\right)
$$




\subsection{Discrete Projection Methods}

We first define the interpolatory projection at $r$ Gauss points.

Let $n \in \mathbb{N}$ and consider the following uniform partition of $[a, b]$ :

$$
a=t_{0}<t_{1}<\cdots<t_{n}=b
$$

Let

$$
h=t_{k}-t_{k-1}=\frac{b-a}{n}, \quad k=1, \ldots, n .
$$

Let $r$ be a positive integer and let

$$
\mathscr{X}_{n}=\left\{g \in L^{\infty}[a, b]:\left.g\right|_{\left[t_{k-1}, t_{k}\right]} \text { is a polynomial of degree } \leq r-1, k=1, \ldots, n\right\}
$$

Let

$$
0<q_{1}<\cdots<q_{r}<1
$$

denote the Gauss-Legendre zeros of order $r$ in $[0,1]$. The collocation nodes are chosen as follows:

$$
\tau_{i}^{k}=t_{k-1}+q_{i} h, \quad i=1, \ldots, r, \quad k=1, \ldots, n .
$$

Define the interpolation operator $Q_{n}: C[a, b] \rightarrow \mathscr{X}_{n}$ as

$$
\left(Q_{n} x\right)\left(\tau_{i}^{k}\right)=x\left(\tau_{i}^{k}\right), \quad i=1, \ldots, r, \quad k=1, \ldots, n
$$

Using the Hahn-Banach extension theorem, as in Atkinson et al [5], $Q_{n}$ can be extended to $L^{\infty}[a, b]$. Note that for $x \in C[a, b], Q_{n} x \rightarrow x$ as $n \rightarrow \infty$. As a consequence,

$$
\sup _{n}\left\|\left.Q_{n}\right|_{C[a, b]}\right\| \leq q
$$

Let

$$
p \in \mathbb{N} \text { and } m=p n \text {. }
$$

Replacing the operator $\mathscr{K}$ by the Nyström operator $\mathscr{K}_{m}$ from (2.6) in (1.3)-(1.7) we obtain discrete versions of various projection methods as given below.

Discrete Collocation method:

$$
z_{n}^{C}-Q_{n} \mathscr{K}_{m}\left(z_{n}^{C}\right)=Q_{n} f
$$


Discrete Iterated Collocation method:

$$
z_{n}^{S}=\mathscr{K}_{m}\left(z_{n}^{C}\right)+f
$$

The discrete modified projection operator is defined as

$$
\tilde{\mathscr{K}}_{n}^{M}(x)=Q_{n} \mathscr{K}_{m}(x)+\mathscr{K}_{m}\left(Q_{n} x\right)-Q_{n} \mathscr{K}_{m}\left(Q_{n} x\right)
$$

Discrete Modified Projection Method: We later show that for $n$ and $m$ big enough,

$$
x_{n}-\tilde{\mathscr{K}}_{n}^{M}\left(x_{n}\right)=f
$$

has a unique solution $z_{n}^{M}$ in a neighbourhood of the exact solution $\varphi$.

Discrete Iterated Modified Projection Method:

$$
\tilde{z}_{n}^{M}=\mathscr{K}_{m}\left(z_{n}^{M}\right)+f
$$

Our aim is to show that $z_{n}^{M} \rightarrow \varphi, \tilde{z}_{n}^{M} \rightarrow \varphi$ and obtain their orders of convergence. We first consider the case of a linear integral operator. The results proved for the linear integral equation are needed in the case of the Urysohn integral equation.

\section{$3 \quad$ Linear Integral Equations}

Let $\kappa(\cdot, \cdot) \in C([a, b] \times[a, b])$. Then the integral operator

$$
(\mathscr{K} x)(s)=\int_{a}^{b} \kappa(s, t) x(t) d t, \quad s \in[a, b]
$$

is a compact linear operator from $L^{\infty}[a, b]$ to $C[a, b]$. Assume that 1 is in the resolvent set of $\mathscr{K}$. Then for $f \in C[a, b]$, the following Fredholm integral equation

$$
x-\mathscr{K} x=f
$$

has a unique solution in $C[a, b]$, say $\varphi$.

In this special case, the Nyström operator defined in (2.6) is given by

$$
\left(\mathscr{K}_{m} x\right)(s)=\tilde{h} \sum_{j=1}^{m} \sum_{i=1}^{\rho} w_{i} \kappa\left(s, \zeta_{i}^{j}\right) x\left(\zeta_{i}^{j}\right), \quad s \in[a, b], \quad x \in C[a, b] .
$$


Note that for $x \in C[a, b], \mathscr{K}_{m} x$ converges to $\mathscr{K} x$ and $\left\{\mathscr{K}_{m}\right\}$ is a collectively compact family. Hence for all $m$ big enough, 1 is in the resolvent set of $\mathscr{K}_{m}$ and the following Nyström approximation

$$
x_{m}-\mathscr{K}_{m} x_{m}=f
$$

has a unique solution, say $\varphi_{m}$. Also,

$$
\left\|\left(I-\mathscr{K}_{m}\right)^{-1}\right\| \leq C_{2} .
$$

If the kernel $\kappa \in C^{d}([a, b] \times[a, b])$ and if the right hand side $f \in C^{d}[a, b]$, then the exact solution $\varphi$ of $(3.1)$ is in $C^{d}[a, b]$ and

$$
\left\|\varphi-\varphi_{m}\right\|_{\infty}=O\left(\tilde{h}^{d}\right)
$$

(See Atkinson [3]).

\subsection{Discrete Modified Projection Methods}

Note that since $\mathscr{K}_{m}$ is a linear operator, the discrete modified projection operator defined by (2.20) can be written as

$$
\tilde{\mathscr{K}}_{n}^{M}=Q_{n} \mathscr{K}_{m}+\mathscr{K}_{m} Q_{n}-Q_{n} \mathscr{K}_{m} Q_{n}
$$

and

$$
\left\|\mathscr{K}_{m}-\tilde{\mathscr{K}}_{n}^{M}\right\|=\left\|\left(I-Q_{n}\right) \mathscr{K}_{m}\left(I-Q_{n}\right)\right\| .
$$

Since $Q_{n}$ converges to the Identity operator pointwise on $C[a, b]$ and $\left\{\mathscr{K}_{m}\right\}$ is a collectively compact family, it follows that

$$
\left\|\left(I-Q_{n}\right) \mathscr{K}_{m}\right\| \rightarrow 0 \text { as } n \rightarrow \infty .
$$

Hence using the estimate (2.17), we obtain

$$
\left\|\mathscr{K}_{m}-\tilde{\mathscr{K}}_{n}^{M}\right\| \leq(1+q)\left\|\left(I-Q_{n}\right) \mathscr{K}_{m}\right\| \rightarrow 0 \text { as } n \rightarrow \infty .
$$


Thus, for $n$ and $m$ big enough, 1 belongs to the resolvent set of $\tilde{\mathscr{K}}_{n}^{M}$ and

$$
x_{n}-\tilde{\mathscr{K}}_{n}^{M} x_{n}=f
$$

has a unique solution, say $z_{n}^{M}$. Also,

$$
\left\|\left(I-\tilde{\mathscr{K}}_{n}^{M}\right)^{-1}\right\| \leq 2 C_{2}
$$

The Discrete Iterated Modified Projection solution is defined as

$$
\tilde{z}_{n}^{M}=\mathscr{K}_{m} z_{n}^{M}+f
$$

\subsection{Orders of Convergence}

Let $p \in \mathbb{N}$ and choose $m=n p$. Then $p \tilde{h}=h$. Note that the $m \rho=n p \rho$ nodes in the composite quadrature rule (2.4) can be divided into $n$ groups of $p \rho$ nodes and each of the $p \rho$ nodes in a group lie precisely in one of the subinterval of the partition (2.13). Based on this observation, we rewrite the expression (3.2) for the Nyström operator $\mathscr{K}_{m}$ as follows.

$$
\left(\mathscr{K}_{m} x\right)(s)=\tilde{h} \sum_{k=1}^{n} \sum_{\nu=1}^{p} \sum_{i=1}^{\rho} w_{i} \kappa\left(s, \zeta_{i}^{(k-1) p+\nu}\right) x\left(\zeta_{i}^{(k-1) p+\nu}\right), \quad s \in[a, b] .
$$

Let

$$
\Psi(t)=\left(t-q_{1}\right) \cdots\left(t-q_{r}\right), \quad t \in[0,1]
$$

where $q_{1}, \ldots, q_{r}$ are Gauss-Legendre zeros in $[0,1]$. Then

$$
\int_{0}^{1} t^{j} \Psi(t) d t=\int_{0}^{1} t^{j}\left(t-q_{1}\right) \cdots\left(t-q_{r}\right)=0 \quad \text { for } \quad 0 \leq j \leq r-1 .
$$

We prove below a discrete analogue of the above result when the integral is replaced by a composite numerical quadrature.

Lemma 3.1. Let $\left\{w_{i}, i=1, \ldots, \rho\right\}$ and $\left\{\mu_{i}, i=1, \ldots, \rho\right\}$ be respectively the weights and the node points in the basic quadrature formula defined in (2.2). Then

$$
\sum_{\nu=1}^{p} \sum_{i=1}^{\rho} w_{i}\left(\frac{\nu-1+\mu_{i}}{p}\right)^{j} \Psi\left(\frac{\nu-1+\mu_{i}}{p}\right)=0, \quad 0 \leq j \leq r-1 .
$$


Proof. Note that

$$
\int_{0}^{1} f(t) d t=\sum_{\nu=1}^{p} \int_{\frac{\nu-1}{p}}^{\frac{\nu}{p}} f(t) d t \approx \frac{1}{p} \sum_{\nu=1}^{p} \sum_{i=1}^{\rho} w_{i} f\left(\frac{\nu-1+\mu_{i}}{p}\right) .
$$

The quadrature rule (2.2) is assumed to be exact for polynomials of degree $\leq 2 r-1$. Hence

$$
\int_{0}^{1} t^{j} \Psi(t) d t=\frac{1}{p} \sum_{\nu=1}^{p} \sum_{i=1}^{\rho} w_{i}\left(\frac{\nu-1+\mu_{i}}{p}\right)^{j} \Psi\left(\frac{\nu-1+\mu_{i}}{p}\right), \quad 0 \leq j \leq r-1 .
$$

Since for $0 \leq j \leq r-1$,

$$
\int_{0}^{1} t^{j} \Psi(t) d t=0
$$

the desired result follows.

For future reference, we quote the following interpolation error estimates from Conte-deBoor [8]. For $t \in\left[t_{k-1}, t_{k}\right]$,

$$
x(t)-\left(Q_{n} x\right)(t)=x\left[\tau_{1}^{k}, \ldots, \tau_{r}^{k}, t\right]\left(t-\tau_{1}^{k}\right) \cdots\left(t-\tau_{r}^{k}\right),
$$

where $x\left[\tau_{1}^{k}, \ldots, \tau_{r}^{k}, t\right]$ denotes the divided difference of $x$ based on $\left\{\tau_{1}^{k}, \ldots, \tau_{r}^{k}, t\right\}$.

Substituting for $\tau_{i}^{k}$ from (2.15) we obtain,

$$
\begin{aligned}
x(t)-\left(Q_{n} x\right)(t) & =x\left[\tau_{1}^{k}, \ldots, \tau_{r}^{k}, t\right]\left(t-t_{k-1}-q_{1} h\right) \cdots\left(t-t_{k-1}-q_{r} h\right) \\
& =x\left[\tau_{1}^{k}, \ldots, \tau_{r}^{k}, t\right]\left(\frac{t-t_{k-1}}{h}-q_{1}\right) \cdots\left(\frac{t-t_{k-1}}{h}-q_{r}\right) h^{r} \\
& =x\left[\tau_{1}^{k}, \ldots, \tau_{r}^{k}, t\right] \Psi\left(\frac{t-t_{k-1}}{h}\right) h^{r} .
\end{aligned}
$$

If $x \in C^{r}[a, b]$, then

$$
\left\|x-Q_{n} x\right\|_{\infty} \leq \frac{\left\|x^{(r)}\right\|_{\infty}}{r !}\|\Psi\|_{\infty} h^{r}=C_{3}\left\|x^{(r)}\right\|_{\infty} h^{r}
$$

where

$$
C_{3}=\frac{\|\Psi\|_{\infty}}{r !}
$$

We use the following notation:

$$
\|\kappa\|_{2 r, \infty}=\max \left\{\left\|D^{(i, j)} \kappa\right\|_{\infty}: 0 \leq i+j \leq 2 r\right\} \text { and }\|x\|_{2 r, \infty}=\max \left\{\left\|x^{(j)}\right\|_{\infty}: 0 \leq j \leq 2 r\right\},
$$


where $x^{(j)}$ denotes the $j$ th derivative of $x$ and

$$
D^{(i, j)} \kappa(s, t)=\frac{\partial^{i+j} \kappa}{\partial s^{i} \partial t^{j}}(s, t), \quad a \leq s, t \leq b .
$$

We now prove some preliminary results which are needed to obtain the orders of convergence in the Discrete Modified ProjectionMethod and the Discrete Iterated Modifited Projection Method. The following proposition is crucial in what follows.

Proposition 3.2. If $\kappa \in C^{r}([a, b] \times[a, b])$ and $x \in C^{2 r}[a, b]$, then

$$
\left\|\mathscr{K}_{m}\left(I-Q_{n}\right) x\right\|_{\infty} \leq C_{4}\|\kappa\|_{r, \infty}\|x\|_{2 r, \infty} h^{2 r}
$$

where

$$
C_{4}=\frac{1}{r !} 2^{r}(b-a)\|\Psi\|_{\infty}\left(\sum_{i=1}^{\rho}\left|w_{i}\right|\right)
$$

is a constant independent of $n$ and of $m$.

Proof. For $s \in[a, b]$,

$$
\begin{aligned}
\left(\mathscr{K}_{m}\left(I-Q_{n}\right) x\right)(s)=\tilde{h} \sum_{k=1}^{n} \sum_{\nu=1}^{p} \sum_{i=1}^{\rho} w_{i} \kappa\left(s, \zeta_{i}^{(k-1) p+\nu}\right) \times & \\
& {\left[x\left(\zeta_{i}^{(k-1) p+\nu}\right)-Q_{n} x\left(\zeta_{i}^{(k-1) p+\nu}\right)\right] . }
\end{aligned}
$$

Substituting from the relation (3.7), we obtain

$$
\begin{aligned}
&\left(\mathscr{K}_{m}\left(I-Q_{n}\right) x\right)(s)=\tilde{h} h^{r} \sum_{k=1}^{n} \sum_{\nu=1}^{p} \sum_{i=1}^{\rho} w_{i} \kappa\left(s, \zeta_{i}^{(k-1) p+\nu}\right) \times \\
& x\left[\tau_{1}^{k}, \ldots, \tau_{r}^{k}, \zeta_{i}^{(k-1) p+\nu}\right] \Psi\left(\frac{\zeta_{i}^{(k-1) p+\nu}-t_{k-1}}{h}\right) .
\end{aligned}
$$

Note that

$$
\frac{\zeta_{i}^{(k-1) p+\nu}-t_{k-1}}{h}=\frac{\left(\nu-1+\mu_{i}\right) \tilde{h}}{p \tilde{h}}=\frac{\nu-1+\mu_{i}}{p} .
$$

Thus,

$$
\begin{aligned}
\left(\mathscr{K}_{m}\left(I-Q_{n}\right) x\right)(s)=\tilde{h} h^{r} \sum_{k=1}^{n} \sum_{\nu=1}^{p} \sum_{i=1}^{\rho} w_{i} \kappa\left(s, \zeta_{i}^{(k-1) p+\nu}\right) \times \\
x\left[\tau_{1}^{k}, \ldots, \tau_{r}^{k}, \zeta_{i}^{(k-1) p+\nu}\right] \Psi\left(\frac{\nu-1+\mu_{i}}{p}\right) .
\end{aligned}
$$


For a fixed $s \in[a, b]$, define

$$
g_{s}^{k}(t)=\kappa(s, t) x\left[\tau_{1}^{k}, \ldots, \tau_{r}^{k}, t\right] \text { for } t_{k-1} \leq t \leq t_{k}
$$

Then

$$
\left(\mathscr{K}_{m}\left(I-Q_{n}\right) x\right)(s)=\tilde{h} h^{r} \sum_{k=1}^{n} \sum_{\nu=1}^{p} \sum_{i=1}^{\rho} w_{i} g_{s}^{k}\left(\zeta_{i}^{(k-1) p+\nu}\right) \Psi\left(\frac{\nu-1+\mu_{i}}{p}\right) .
$$

Since $k \in C^{r}([a, b] \times[a, b])$ and $x \in C^{2 r}[a, b]$, it follows that $g_{s}^{k} \in C^{r}\left[t_{k-1}, t_{k}\right]$ for $k=1, \ldots, n$. For $t \in\left[t_{k-1}, t_{k}\right]$, we expand $g_{s}^{k}(t)$ about $t_{k-1}$ and obtain

$$
g_{s}^{k}(t)=\sum_{j=0}^{r-1} \frac{1}{j !}\left(t-t_{k-1}\right)^{j}\left(g_{s}^{k}\right)^{(j)}\left(t_{k-1}\right)+\frac{1}{r !}\left(t-t_{k-1}\right)^{r}\left(g_{s}^{k}\right)^{(r)}\left(\xi_{t}^{k}\right), \quad \xi_{t}^{k} \in\left(t_{k-1}, t_{k}\right) .
$$

Substitute the above expression for $g_{s}^{k}(t)$ in (3.12) to obtain

$$
\begin{aligned}
\left(\mathscr{K}_{m}\left(I-Q_{n}\right) x\right)(s) & =\tilde{h} h^{r} \sum_{k=1}^{n} \sum_{j=0}^{r-1} \frac{1}{j !}\left(g_{s}^{k}\right)^{(j)}\left(t_{k-1}\right) \times \\
& \sum_{\nu=1}^{p} \sum_{i=1}^{\rho} w_{i}\left(\zeta_{i}^{(k-1) p+\nu}-t_{k-1}\right)^{j} \Psi\left(\frac{\nu-1+\mu_{i}}{p}\right) \\
& +\frac{1}{r !} \tilde{h} h^{r} \sum_{k=1}^{n} \sum_{\nu=1}^{p} \sum_{i=1}^{\rho} w_{i}\left(\zeta_{i}^{(k-1) p+\nu}-t_{k-1}\right)^{r}\left(g_{s}^{k}\right)^{(r)}\left(\xi_{i, \nu}^{k}\right) \Psi\left(\frac{\nu-1+\mu_{i}}{p}\right) .
\end{aligned}
$$

Using (3.10) the above equation reduces to

$$
\begin{aligned}
\left(\mathscr{K}_{m}\left(I-Q_{n}\right) x\right)(s) & =\tilde{h} h^{r} \sum_{k=1}^{n} \sum_{j=0}^{r-1} \frac{1}{j !}\left(g_{s}^{k}\right)^{(j)}\left(t_{k-1}\right) \tilde{h}^{j} \times \\
& \sum_{\nu=1}^{p} \sum_{i=1}^{\rho} w_{i}\left(\nu-1+\mu_{i}\right)^{j} \Psi\left(\frac{\nu-1+\mu_{i}}{p}\right) \\
& +\frac{1}{r !} \tilde{h}^{r+1} h^{r} \sum_{k=1}^{n} \sum_{\nu=1}^{p} \sum_{i=1}^{\rho} w_{i}\left(\nu-1+\mu_{i}\right)^{r}\left(g_{s}^{k}\right)^{(r)}\left(\xi_{i, \nu}^{k}\right) \Psi\left(\frac{\nu-1+\mu_{i}}{p}\right) .
\end{aligned}
$$

From Lemma 3.1 we see that the first term in the above expression vanishes. Hence

$$
\begin{aligned}
\left\|\mathscr{K}_{m}\left(I-Q_{n}\right) x\right\|_{\infty} \leq \frac{1}{r !} \tilde{h}^{r+1} h^{r} n \max _{s \in[a, b]} \max _{1 \leq k \leq n}\left(\max _{t \in\left[t_{k-1}, t_{k}\right]}\left|\left(g_{s}^{k}\right)^{(r)}(t)\right|\right) \times \\
\sum_{\nu=1}^{p} \sum_{i=1}^{\rho}\left\{\left|w_{i}\right|\left|\nu-1+\mu_{i}\right|^{r}\left|\Psi\left(\frac{\nu-1+\mu_{i}}{p}\right)\right|\right\} .
\end{aligned}
$$

It can be seen that for $s \in[a, b]$,

$$
\max _{t \in\left[t_{k-1}, t_{k}\right]}\left|\left(g_{s}^{k}\right)^{(r)}(t)\right| \leq 2^{r}\|\kappa\|_{r, \infty}\|x\|_{2 r, \infty}
$$


Since $\mu_{i} \in[0,1]$, it follows that

$$
\nu-1+\mu_{i} \leq p \text { for } 1 \leq \nu \leq p
$$

and hence

$$
\sum_{\nu=1}^{p} \sum_{i=1}^{\rho}\left\{\left|w_{i}\right|\left|\nu-1+\mu_{i}\right|^{r}\left|\Psi\left(\frac{\nu-1+\mu_{i}}{p}\right)\right|\right\} \leq p^{r+1}\|\Psi\|_{\infty}\left(\sum_{i=1}^{\rho}\left|w_{i}\right|\right) .
$$

Since $\tilde{h} p=h$ and $h n=b-a$, it follows that

$$
\left\|\mathscr{K}_{m}\left(I-Q_{n}\right) x\right\|_{\infty} \leq C_{4}\|\kappa\|_{r, \infty}\|x\|_{2 r, \infty} h^{2 r}
$$

which completes the proof.

The following two results are proved using Proposition 3.2 .

Proposition 3.3. If $\kappa \in C^{2 r}([a, b] \times[a, b])$, then

$$
\left\|\mathscr{K}_{m}\left(I-Q_{n}\right) \mathscr{K}_{m}\right\| \leq C_{5}\|\kappa\|_{r, \infty}\|\kappa\|_{2 r, \infty} h^{2 r},
$$

where

$$
C_{5}=C_{4}(b-a)\left(\sum_{i=1}^{\rho}\left|w_{i}\right|\right)=\frac{1}{r !} 2^{r}(b-a)^{2}\|\Psi\|_{\infty}\left(\sum_{i=1}^{\rho}\left|w_{i}\right|\right)^{2}
$$

is a constant independent of $n$ and of $m$.

Proof. From Proposition 3.2, we have

$$
\left\|\mathscr{K}_{m}\left(I-Q_{n}\right) \mathscr{K}_{m} x\right\|_{\infty} \leq C_{4}\|\kappa\|_{r, \infty}\left\|\mathscr{K}_{m} x\right\|_{2 r, \infty} h^{2 r} .
$$

From (3.2)

$$
\left(\mathscr{K}_{m} x\right)(s)=\tilde{h} \sum_{j=1}^{m} \sum_{i=1}^{\rho} w_{i} \kappa\left(s, \zeta_{i}^{j}\right) x\left(\zeta_{i}^{j}\right) .
$$

Hence for $\beta=1, \ldots, 2 r$,

$$
\left(\mathscr{K}_{m} x\right)^{(\beta)}(s)=\tilde{h} \sum_{j=1}^{m} \sum_{i=1}^{\rho} w_{i} D^{(\beta, 0)} \kappa\left(s, \zeta_{i}^{j}\right) x\left(\zeta_{i}^{j}\right)
$$

and

$$
\left\|\left(\mathscr{K}_{m} x\right)^{(\beta)}\right\|_{\infty} \leq \tilde{h} m\left(\sum_{i=1}^{\rho}\left|w_{i}\right|\right)\left\|D^{(\beta, 0)} \kappa\right\|_{\infty}\|x\|_{\infty} .
$$


As a consequence,

$$
\left\|\mathscr{K}_{m} x\right\|_{2 r, \infty} \leq(b-a)\left(\sum_{i=1}^{\rho}\left|w_{i}\right|\right)\|\kappa\|_{2 r, \infty}\|x\|_{\infty} .
$$

Thus,

$$
\left\|\mathscr{K}_{m}\left(I-Q_{n}\right) \mathscr{K}_{m} x\right\|_{\infty} \leq C_{4}(b-a)\left(\sum_{i=1}^{\rho}\left|w_{i}\right|\right)\|\kappa\|_{r, \infty}\|\kappa\|_{2 r, \infty}\|x\|_{\infty} h^{2 r}
$$

The desired estimate follows by taking the supremum over the set $\left\{x \in C[a, b]:\|x\|_{\infty} \leq 1\right\}$.

Proposition 3.4. If $\kappa \in C^{2 r}([a, b] \times[a, b])$ and $x \in C^{2 r}[a, b]$, then

$$
\left\|\left(I-Q_{n}\right) \mathscr{K}_{m}\left(I-Q_{n}\right) x\right\|_{\infty} \leq C_{3} C_{4}\|\kappa\|_{2 r, \infty}\|x\|_{2 r, \infty} h^{3 r}
$$

If $\kappa \in C^{3 r}([a, b] \times[a, b])$ and $x \in C^{2 r}[a, b]$, then

$$
\left\|\mathscr{K}_{m}\left(I-Q_{n}\right) \mathscr{K}_{m}\left(I-Q_{n}\right) x\right\|_{\infty} \leq\left(C_{4}\right)^{2}\|\kappa\|_{r, \infty}\|\kappa\|_{3 r, \infty}\|x\|_{2 r, \infty} h^{4 r}
$$

Proof. From the estimate (3.8), we obtain

$$
\left\|\left(I-Q_{n}\right) \mathscr{K}_{m}\left(I-Q_{n}\right) x\right\|_{\infty} \leq C_{3}\left\|\left(\mathscr{K}_{m}\left(I-Q_{n}\right) x\right)^{(r)}\right\|_{\infty} h^{r}
$$

Differentiating (3.11) $r$ times with respect to $s$, we obtain

$$
\begin{aligned}
&\left(\mathscr{K}_{m}\left(I-Q_{n}\right) x\right)^{(r)}(s)=\tilde{h} h^{r} \sum_{k=1}^{n} \sum_{\nu=1}^{p} \sum_{i=1}^{\rho} w_{i} \frac{\partial^{r} \kappa}{\partial s^{r}}\left(s, \zeta_{i}^{(k-1) p+\nu}\right) \times \\
& x\left[\tau_{1}^{k}, \ldots, \tau_{r}^{k}, \zeta_{i}^{(k-1) p+\nu}\right] \Psi\left(\frac{\nu-1+\mu_{i}}{p}\right) .
\end{aligned}
$$

Let

$$
\ell(s, t)=\frac{\partial^{r} \kappa}{\partial s^{r}}(s, t), \quad a \leq s, t \leq b .
$$

If $\kappa \in C^{2 r}([a, b] \times[a, b])$, then $\ell \in C^{r}([a, b] \times[a, b])$. Hence proceeding as in the proof of Proposition 3.2, we obtain

$$
\left\|\left(\mathscr{K}_{m}\left(I-Q_{n}\right) x\right)^{(r)}\right\|_{\infty} \leq C_{4}\|\ell\|_{r, \infty}\|x\|_{2 r, \infty} h^{2 r} \leq C_{4}\|\kappa\|_{2 r, \infty}\|x\|_{2 r, \infty} h^{2 r} .
$$

As a consequence,

$$
\left\|\left(I-Q_{n}\right) \mathscr{K}_{m}\left(I-Q_{n}\right) x\right\|_{\infty} \leq C_{3} C_{4}\|\kappa\|_{2 r, \infty}\|x\|_{2 r, \infty} h^{3 r},
$$


which completes the proof of (3.14).

Let $\kappa \in C^{3 r}([a, b] \times[a, b])$ and $x \in C^{2 r}[a, b]$. Then $\mathscr{K}_{m}\left(I-Q_{n}\right) x \in C^{3 r}[a, b]$. Using (3.9) we obtain,

$$
\left\|\mathscr{K}_{m}\left(I-Q_{n}\right) \mathscr{K}_{m}\left(I-Q_{n}\right) x\right\|_{\infty} \leq C_{4}\|\kappa\|_{r, \infty}\left\|\mathscr{K}_{m}\left(I-Q_{n}\right) x\right\|_{2 r, \infty} h^{2 r}
$$

From (3.11) for $j=0,1, \ldots, 2 r$,

$$
\begin{aligned}
&\left(\mathscr{K}_{m}\left(I-Q_{n}\right) x\right)^{(j)}(s)=\tilde{h} h^{r} \sum_{k=1}^{n} \sum_{\nu=1}^{p} \sum_{i=1}^{\rho} w_{i} \frac{\partial^{j} \kappa}{\partial s^{j}}\left(s, \zeta_{i}^{(k-1) p+\nu}\right) \times \\
& x\left[\tau_{1}^{k}, \ldots, \tau_{r}^{k}, \zeta_{i}^{(k-1) p+\nu}\right] \Psi\left(\frac{\nu-1+\mu_{i}}{p}\right) .
\end{aligned}
$$

Note that $\frac{\partial^{j} \kappa}{\partial s^{j}} \in C^{3 r-j}([a, b] \times[a, b])$, for $j=0,1, \ldots, 2 r$. Proceeding as in the proof of Proposition 3.2, we obtain

$$
\left\|\left(\mathscr{K}_{m}\left(I-Q_{n}\right) x\right)^{(j)}\right\|_{\infty} \leq C_{4}\|\kappa\|_{j+r, \infty}\|x\|_{2 r, \infty} h^{2 r}
$$

and hence

$$
\begin{aligned}
\left\|\mathscr{K}_{m}\left(I-Q_{n}\right) x\right\|_{2 r, \infty} & =\max \left\{\left\|\left(\mathscr{K}_{m}\left(I-Q_{n}\right) x\right)^{(j)}\right\|_{\infty}: 0 \leq j \leq 2 r\right\} \\
& \leq C_{4}\|\kappa\|_{3 r, \infty}\|x\|_{2 r, \infty} h^{2 r} .
\end{aligned}
$$

As a consequence,

$$
\left\|\mathscr{K}_{m}\left(I-Q_{n}\right) \mathscr{K}_{m}\left(I-Q_{n}\right) x\right\|_{\infty} \leq\left(C_{4}\right)^{2}\|\kappa\|_{r, \infty}\|\kappa\|_{3 r, \infty}\|x\|_{2 r, \infty} h^{4 r}
$$

which completes the proof of (3.15).

We now prove our main result for linear integral equations.

Theorem 3.5. If $\kappa \in C^{d}([a, b] \times[a, b])$ and $f \in C^{d}[a, b]$, then

$$
\left\|\varphi-z_{n}^{M}\right\|_{\infty}=O\left(\max \left\{\tilde{h}^{d}, h^{3 r}\right\}\right) .
$$

If $\kappa \in C^{\max \{3 r, d\}}([a, b] \times[a, b])$ and $f \in C^{d}[a, b]$, then

$$
\left\|\varphi-\tilde{z}_{n}^{M}\right\|_{\infty}=O\left(\max \left\{\tilde{h}^{d}, h^{4 r}\right\}\right) .
$$


Proof. Since

$$
\varphi-z_{n}^{M}=(I-\mathscr{K})^{-1} f-\left(I-\tilde{\mathscr{K}}_{n}^{M}\right)^{-1} f=\left(I-\tilde{\mathscr{K}}_{n}^{M}\right)^{-1}\left(\mathscr{K}-\tilde{\mathscr{K}}_{n}^{M}\right) \varphi
$$

using (3.4) we obtain,

$$
\begin{aligned}
\left\|\varphi-z_{n}^{M}\right\|_{\infty} & \leq 2 C_{2}\left(\left\|\mathscr{K} \varphi-\mathscr{K}_{m} \varphi\right\|_{\infty}+\left\|\left(\mathscr{K}_{m}-\tilde{\mathscr{K}}_{n}^{M}\right) \varphi\right\|_{\infty}\right) \\
& \leq 2 C_{2}\left(\left\|\mathscr{K} \varphi-\mathscr{K}_{m} \varphi\right\|_{\infty}+\left\|\left(I-Q_{n}\right) \mathscr{K}_{m}\left(I-Q_{n}\right) \varphi\right\|_{\infty}\right) .
\end{aligned}
$$

Using the estimates (2.9) and (3.14) we obtain,

$$
\left\|\varphi-z_{n}^{M}\right\|_{\infty}=O\left(\max \left\{\tilde{h}^{d}, h^{3 r}\right\}\right)
$$

which completes the proof of (3.17).

Note that

$$
\begin{aligned}
\varphi_{m}-z_{n}^{M} & =\left(I-\mathscr{K}_{m}\right)^{-1}\left(\mathscr{K}_{m}-\tilde{\mathscr{K}}_{n}^{M}\right) z_{n}^{M} \\
& =\left(I-\mathscr{K}_{m}\right)^{-1}\left(I-Q_{n}\right) \mathscr{K}_{m}\left(I-Q_{n}\right) z_{n}^{M} .
\end{aligned}
$$

Hence

$$
\begin{aligned}
\varphi_{m}-\tilde{z}_{n}^{M} & =\mathscr{K}_{m} \varphi_{m}-\mathscr{K}_{m} z_{n}^{M} \\
& =\left(I-\mathscr{K}_{m}\right)^{-1} \mathscr{K}_{m}\left(I-Q_{n}\right) \mathscr{K}_{m}\left(I-Q_{n}\right) z_{n}^{M} \\
& =\left(I-\mathscr{K}_{m}\right)^{-1} \mathscr{K}_{m}\left(I-Q_{n}\right) \mathscr{K}_{m}\left(I-Q_{n}\right)\left(z_{n}^{M}-\varphi\right) \\
& +\left(I-\mathscr{K}_{m}\right)^{-1} \mathscr{K}_{m}\left(I-Q_{n}\right) \mathscr{K}_{m}\left(I-Q_{n}\right) \varphi
\end{aligned}
$$

Thus,

$$
\begin{aligned}
\left\|\varphi_{m}-\tilde{z}_{n}^{M}\right\|_{\infty} & \leq C_{2}\left(1+\left\|Q_{n}\right\|\right)\left\|\mathscr{K}_{m}\left(I-Q_{n}\right) \mathscr{K}_{m}\right\|\left\|z_{n}^{M}-\varphi\right\|_{\infty} \\
& +C_{2}\left\|\mathscr{K}_{m}\left(I-Q_{n}\right) \mathscr{K}_{m}\left(I-Q_{n}\right) \varphi\right\|_{\infty} .
\end{aligned}
$$

Using (3.13), (3.15) and (3.17) and the fact that $d \geq 2 r$, it can be seen that

$$
\left\|\varphi_{m}-\tilde{z}_{n}^{M}\right\|_{\infty}=O\left(h^{4 r}\right)
$$

Hence using (3.3) we obtain,

$$
\left\|\varphi-\tilde{z}_{n}^{M}\right\|_{\infty} \leq\left\|\varphi-\varphi_{m}\right\|_{\infty}+\left\|\varphi_{m}-\tilde{z}_{n}^{M}\right\|_{\infty}=O\left(\max \left\{\tilde{h}^{d}, h^{4 r}\right\}\right),
$$

which completes the proof. 


\section{Discrete Modified Projection Method for Urysohn Integral Equations}

In this section we consider approximation of the Urysohn integral equation (1.1)-(1.2) by the discrete version of the modified projection method. For a fixed $\delta>0$, a closed neighbourhood $B(\varphi, \delta)$ of the exact solution $\varphi$ is defined in (2.11). First we prove a result about the Nyström operator $\mathscr{K}_{m}$ defined in (2.6). Let $\frac{\partial^{2} \kappa}{\partial u^{2}} \in C(\Omega)$ and define

$$
C_{6}=\max _{\substack{s, t \in[a, b] \\|u| \leq\|\varphi\|_{\infty}+\delta}}\left|\frac{\partial^{2} \kappa}{\partial u^{2}}(s, t, u)\right| .
$$

Proposition 4.1. Let $\frac{\partial^{2} \kappa}{\partial u^{2}} \in C(\Omega)$. Then for $v_{1}, v_{2} \in B(\varphi, \delta)$ and for $s \in[a, b]$,

$$
\mathscr{K}_{m}\left(v_{2}\right)(s)-\mathscr{K}_{m}\left(v_{1}\right)(s)-\mathscr{K}_{m}^{\prime}\left(v_{1}\right)\left(v_{2}-v_{1}\right)(s)=R\left(v_{2}-v_{1}\right)(s)
$$

where

$$
\left\|R\left(v_{2}-v_{1}\right)\right\|_{\infty} \leq \frac{C_{6}(b-a)}{2}\left(\sum_{i=1}^{\rho}\left|w_{i}\right|\right)\left\|v_{2}-v_{1}\right\|_{\infty}^{2} .
$$

Proof. If $v_{1}, v_{2} \in B(\varphi, \delta)$, then by the generalized Taylor's theorem,

$$
\mathscr{K}_{m}\left(v_{2}\right)(s)-\mathscr{K}_{m}\left(v_{1}\right)(s)-\mathscr{K}_{m}^{\prime}\left(v_{1}\right)\left(v_{2}-v_{1}\right)(s)=R\left(v_{2}-v_{1}\right)(s), s \in[a, b],
$$

where

$$
R\left(v_{2}-v_{1}\right)(s)=\int_{0}^{1}(1-\theta) \mathscr{K}_{m}^{\prime \prime}\left(v_{1}+\theta\left(v_{2}-v_{1}\right)\right)\left(v_{2}-v_{1}\right)^{2}(s) d \theta
$$

For $s \in[a, b]$ and $\theta \in[0,1]$, define

$$
\begin{aligned}
\left(S_{\theta}\left(v_{2}-v_{1}\right)\right)(s) & =\mathscr{K}_{m}^{\prime \prime}\left(v_{1}+\theta\left(v_{2}-v_{1}\right)\right)\left(v_{2}-v_{1}\right)^{2}(s) \\
& =\tilde{h} \sum_{j=1}^{m} \sum_{i=1}^{\rho} w_{i} \frac{\partial^{2} \kappa}{\partial u^{2}}\left(s, \zeta_{i}^{j}, v_{1}\left(\zeta_{i}^{j}\right)+\theta\left(v_{2}-v_{1}\right)\left(\zeta_{i}^{j}\right)\right)\left(v_{2}-v_{1}\right)^{2}\left(\zeta_{i}^{j}\right)
\end{aligned}
$$

Then

$$
\left\|S_{\theta}\left(v_{2}-v_{1}\right)\right\|_{\infty} \leq C_{6}(b-a)\left(\sum_{i=1}^{\rho}\left|w_{i}\right|\right)\left\|v_{2}-v_{1}\right\|_{\infty}^{2}
$$


Since

$$
R\left(v_{2}-v_{1}\right)(s)=\int_{0}^{1}(1-\theta) S_{\theta}\left(v_{2}-v_{1}\right)(s) d \theta
$$

it follows that

$$
\left\|R\left(v_{2}-v_{1}\right)\right\|_{\infty} \leq \frac{C_{6}(b-a)}{2}\left(\sum_{i=1}^{\rho}\left|w_{i}\right|\right)\left\|v_{2}-v_{1}\right\|_{\infty}^{2} .
$$

This completes the proof.

Remark 4.2. Note that

$$
\mathscr{K}^{\prime}(\varphi) v(s)=\int_{a}^{b} \frac{\partial \kappa}{\partial u}(s, t, \varphi(t)) v(t) d t
$$

whereas

$$
\mathscr{K}_{m}^{\prime}(\varphi) v(s)=\tilde{h} \sum_{j=1}^{m} \sum_{i=1}^{\rho} w_{i} \frac{\partial \kappa}{\partial u}\left(s, \zeta_{i}^{j}, \varphi\left(\zeta_{i}^{j}\right)\right) v\left(\zeta_{i}^{j}\right), \quad s \in[a, b] .
$$

Thus, $\mathscr{K}_{m}^{\prime}(\varphi)$ is the Nyström approximation of the linear operator $\mathscr{K}^{\prime}(\varphi)$ associated with a convergent quadrature formula. Hence $\mathscr{K}_{m}^{\prime}(\varphi)$ converges to $\mathscr{K}^{\prime}(\varphi)$ pointwise and $\mathscr{K}_{m}^{\prime}(\varphi)$ is a collectively compact family.

As before, now onwards we assume that

$$
m=n p \quad \text { for some } p \in \mathbb{N} \text {. }
$$

It follows that

$$
\left\|\left(I-Q_{n}\right) \mathscr{K}_{m}^{\prime}(\varphi)\right\| \rightarrow 0 \text { as } n \rightarrow \infty .
$$

It is assumed that $\left(I-\mathscr{K}^{\prime}(\varphi)\right)^{-1}: C[a, b] \rightarrow C[a, b]$ is a bounded linear operator. Hence there exists a positive integer $m_{1} \geq m_{0}$ such that for $m \geq m_{1},\left(I-\mathscr{K}_{m}^{\prime}(\varphi)\right)^{-1}$ exists and

$$
\left\|\left(I-\mathscr{K}_{m}^{\prime}(\varphi)\right)^{-1}\right\| \leq C_{7}
$$

See Atkinson [3].

We prove some preliminary results which are needed to obtain the order of convergence of the discrete modified projection solution $z_{n}^{M}$. 
Proposition 4.3. Let $\frac{\partial^{2} \kappa}{\partial u^{2}} \in C(\Omega)$. Then $\mathscr{K}_{m}^{\prime}$ is Lipschitz continuous in $B(\varphi, \delta)$ :

$$
\left\|\mathscr{K}_{m}^{\prime}(x)-\mathscr{K}_{m}^{\prime}(y)\right\| \leq \gamma\|x-y\|_{\infty}, \quad x, y \in B(\varphi, \delta)
$$

where $\gamma$ is a constant independent of $m$.

Proof. For $x, y \in B(\varphi, \delta)$,

$$
\left\|\mathscr{K}_{m}^{\prime}(x)-\mathscr{K}_{m}^{\prime}(y)\right\|=\sup _{\|v\| \leq 1} \sup _{s \in[a, b]}\left|\left(\mathscr{K}_{m}^{\prime}(x)-\mathscr{K}_{m}^{\prime}(y)\right) v(s)\right|
$$

For $s \in[a, b]$, we have

$$
\left(\mathscr{K}_{m}^{\prime}(x)-\mathscr{K}_{m}^{\prime}(y)\right) v(s)=\tilde{h} \sum_{j=1}^{m} \sum_{i=1}^{\rho} w_{i}\left(\frac{\partial \kappa}{\partial u}\left(s, \zeta_{i}^{j}, x\left(\zeta_{i}^{j}\right)\right)-\frac{\partial \kappa}{\partial u}\left(s, \zeta_{i}^{j}, y\left(\zeta_{i}^{j}\right)\right)\right) v\left(\zeta_{i}^{j}\right) .
$$

By the Mean Value Theorem,

$$
\left(\mathscr{K}_{m}^{\prime}(x)-\mathscr{K}_{m}^{\prime}(y)\right) v(s)=\tilde{h} \sum_{j=1}^{m} \sum_{i=1}^{\rho} w_{i} \frac{\partial^{2} \kappa}{\partial u^{2}}\left(s, \zeta_{i}^{j}, \eta_{i}^{j}\right)\left(x\left(\zeta_{i}^{j}\right)-y\left(\zeta_{i}^{j}\right)\right) v\left(\zeta_{i}^{j}\right)
$$

where

$$
\eta_{i}^{j}=\theta_{i}^{j} x\left(\zeta_{i}^{j}\right)+\left(1-\theta_{i}^{j}\right) y\left(\zeta_{i}^{j}\right) \text { for some } \theta_{i}^{j} \in(0,1)
$$

Since $x, y \in B(\varphi, \delta)$, it follows that

$$
\left|\eta_{i}^{j}\right| \leq\|\varphi\|_{\infty}+\delta, \quad i=1, \ldots, \rho, j=1, \ldots, m
$$

Hence for $s \in[a, b]$,

$$
\begin{aligned}
\left|\left(\mathscr{K}_{m}^{\prime}(x)-\mathscr{K}_{m}^{\prime}(y)\right) v(s)\right| & \leq C_{6} \tilde{h} m\left(\sum_{i=1}^{\rho}\left|w_{i}\right|\right)\|x-y\|_{\infty}\|v\|_{\infty} \\
& =\gamma\|x-y\|_{\infty}\|v\|_{\infty},
\end{aligned}
$$

where

$$
\gamma=C_{6}(b-a)\left(\sum_{i=1}^{\rho}\left|w_{i}\right|\right)
$$

$C_{6}$ being defined in (4.1). Hence

$$
\left\|\left(\mathscr{K}_{m}^{\prime}(x)-\mathscr{K}_{m}^{\prime}(y)\right) v\right\|_{\infty} \leq \gamma\|x-y\|_{\infty}\|v\|_{\infty}
$$

Taking the supremum over the set $\left\{v \in C[a, b]:\|v\|_{\infty} \leq 1\right\}$, the required result follows. 
Proposition 4.4. Let $\frac{\partial^{2} \kappa}{\partial u^{2}} \in C(\Omega)$. There exists a positive integer $n_{1}$ such that for $n \geq n_{1}$ and for $m \geq m_{1}, I-\left(\tilde{\mathscr{K}}_{n}^{M}\right)^{\prime}(\varphi)$ is invertible and

$$
\left\|\left(I-\left(\tilde{\mathscr{K}}_{n}^{M}\right)^{\prime}(\varphi)\right)^{-1}\right\| \leq 2 C_{7} .
$$

Proof. Fix $m=n p \geq m_{1}$. Then by (4.7)

$$
\left\|\left(I-\mathscr{K}_{m}^{\prime}(\varphi)\right)^{-1}\right\| \leq C_{7}
$$

From the definition of $\tilde{\mathscr{K}}_{n}^{M}(x)$ in (2.20), it follows that

$$
\left(\tilde{\mathscr{K}}_{n}^{M}\right)^{\prime}(x)=Q_{n} \mathscr{K}_{m}^{\prime}(x)+\left(I-Q_{n}\right) \mathscr{K}_{m}^{\prime}\left(Q_{n} x\right) Q_{n}
$$

Hence

$$
\begin{aligned}
\mathscr{K}_{m}^{\prime}(\varphi)-\left(\tilde{\mathscr{K}}_{n}^{M}\right)^{\prime}(\varphi) & =\left(I-Q_{n}\right) \mathscr{K}_{m}^{\prime}(\varphi)-\left(I-Q_{n}\right) \mathscr{K}_{m}^{\prime}\left(Q_{n} \varphi\right) Q_{n} \\
& =\left(I-Q_{n}\right) \mathscr{K}_{m}^{\prime}(\varphi)\left(I-Q_{n}\right)+\left(I-Q_{n}\right)\left(\mathscr{K}_{m}^{\prime}(\varphi)-\mathscr{K}_{m}^{\prime}\left(Q_{n} \varphi\right)\right) Q_{n}
\end{aligned}
$$

Since $\varphi \in C[a, b]$, it follows that

$$
\left\|Q_{n} \varphi-\varphi\right\|_{\infty} \rightarrow 0 \text { as } n \rightarrow \infty
$$

Hence there exists a positive integer $n_{0}$ such that

$$
n \geq n_{0} \Rightarrow Q_{n} \varphi \in B(\varphi, \delta)
$$

By Proposition 4.3,

$$
\left\|\mathscr{K}_{m}^{\prime}(\varphi)-\mathscr{K}_{m}^{\prime}\left(Q_{n} \varphi\right)\right\| \leq \gamma\left\|\varphi-Q_{n} \varphi\right\|_{\infty} \rightarrow 0
$$

and by (4.6)

$$
\left\|\left(I-Q_{n}\right) \mathscr{K}_{m}^{\prime}(\varphi)\right\| \rightarrow 0 \text { as } n \rightarrow \infty
$$

Hence for $n \geq n_{0}$,

$$
\begin{aligned}
\left\|\mathscr{K}_{m}^{\prime}(\varphi)-\left(\tilde{\mathscr{K}}_{n}^{M}\right)^{\prime}(\varphi)\right\| & \leq\left(1+\left\|Q_{n}\right\|\right)\left\|\left(I-Q_{n}\right) \mathscr{K}_{m}^{\prime}(\varphi)\right\| \\
& +\left\|Q_{n}\right\|\left(1+\left\|Q_{n}\right\|\right)\left\|\mathscr{K}_{m}^{\prime}(\varphi)-\mathscr{K}_{m}^{\prime}\left(Q_{n} \varphi\right)\right\| \\
& \leq(1+q)\left\|\left(I-Q_{n}\right) \mathscr{K}_{m}^{\prime}(\varphi)\right\|+q(1+q) \gamma\left\|\varphi-Q_{n} \varphi\right\|_{\infty} .
\end{aligned}
$$


Thus,

$$
\left\|\mathscr{K}_{m}^{\prime}(\varphi)-\left(\tilde{\mathscr{K}}_{n}^{M}\right)^{\prime}(\varphi)\right\| \rightarrow 0 \text { as } n \rightarrow \infty
$$

Choose $n_{1} \geq n_{0}$ such that

$$
n \geq n_{1} \Rightarrow\left\|\left(\tilde{\mathscr{K}}_{n}^{M}\right)^{\prime}(\varphi)-\mathscr{K}_{m}^{\prime}(\varphi)\right\| \leq \frac{1}{2 C_{7}}
$$

Since

$$
I-\left(\tilde{\mathscr{K}}_{n}^{M}\right)^{\prime}(\varphi)=\left[I-\left\{\left(\tilde{\mathscr{K}}_{n}^{M}\right)^{\prime}(\varphi)-\mathscr{K}_{m}^{\prime}(\varphi)\right\}\left(I-\mathscr{K}_{m}^{\prime}(\varphi)\right)^{-1}\right]\left(I-\mathscr{K}_{m}^{\prime}(\varphi)\right)
$$

it follows that for $n \geq n_{1}$,

$$
\left\|\left(I-\left(\tilde{\mathscr{K}}_{n}^{M}\right)^{\prime}(\varphi)\right)^{-1}\right\| \leq 2\left\|\left(I-\mathscr{K}_{m}^{\prime}(\varphi)\right)^{-1}\right\| \leq 2 C_{7} .
$$

This completes the proof.

Remark 4.5. For $n \geq n_{1}$ and $m \geq m_{1}$, define

$$
B_{n}(x)=x-\left[I-\left(\tilde{\mathscr{K}}_{n}^{M}\right)^{\prime}(\varphi)\right]^{-1}\left\{x-\tilde{\mathscr{K}}_{n}^{M}(x)-f\right\}
$$

Then

$$
B_{n}(x)=x \text { if and only if } x-\tilde{\mathscr{K}}_{n}^{M}(x)=f .
$$

As in Grammont [10, it can be shown that there is a $\delta_{0}>0$ such that $B_{n}$ has a unique fixed point $z_{n}^{M}$ in $B\left(\varphi, \delta_{0}\right)$ and that

$$
\left\|z_{n}^{M}-\varphi\right\|_{\infty} \leq \frac{3}{2}\left\|\left[I-\left(\tilde{\mathscr{K}}_{n}^{M}\right)^{\prime}(\varphi)\right]^{-1}\left[\mathscr{K}(\varphi)-\tilde{\mathscr{K}}_{n}^{M}(\varphi)\right]\right\|_{\infty} .
$$

Hence

$$
\left\|z_{n}^{M}-\varphi\right\|_{\infty} \leq 3 C_{7}\left(\left\|\mathscr{K}(\varphi)-\mathscr{K}_{m}(\varphi)\right\|_{\infty}+\left\|\mathscr{K}_{m}(\varphi)-\tilde{\mathscr{K}}_{n}^{M}(\varphi)\right\|_{\infty}\right) .
$$

Without loss of generality, assume that

$$
n \geq n_{1} \Rightarrow Q_{n} \varphi \in B\left(\varphi, \delta_{0}\right) \text { and } n \geq n_{1}, m \geq m_{1}=n_{1} p \Rightarrow \varphi_{m} \in B\left(\varphi, \delta_{0}\right), Q_{n} \varphi_{m} \in B\left(\varphi, \delta_{0}\right) .
$$


By (2.9),

$$
\left\|\mathscr{K}(\varphi)-\mathscr{K}_{m}(\varphi)\right\|_{\infty}=O\left(\tilde{h}^{d}\right)
$$

In order to obtain the order of convergence for the term $\left\|\mathscr{K}_{m}(\varphi)-\tilde{\mathscr{K}}_{n}^{M}(\varphi)\right\|_{\infty}$ in the estimate (4.12), we prove the following result.

Proposition 4.6. Let $r \geq 1$. If $\frac{\partial^{2} \kappa}{\partial u^{2}} \in C^{r}(\Omega)$ and $f \in C^{r}[a, b]$, then for $n \geq n_{1}$,

$$
\left\|\left(I-Q_{n}\right)\left(\mathscr{K}_{m}\left(Q_{n} \varphi\right)-\mathscr{K}_{m}(\varphi)-\mathscr{K}_{m}^{\prime}(\varphi)\left(Q_{n} \varphi-\varphi\right)\right)\right\|_{\infty}=O\left(h^{3 r}\right) .
$$

Proof. Let $\delta=\delta_{0}, v_{1}=\varphi$ and $v_{2}=Q_{n} \varphi$ in Proposition 4.1. Then

$$
\mathscr{K}_{m}\left(Q_{n} \varphi\right)-\mathscr{K}_{m}(\varphi)-\mathscr{K}_{m}^{\prime}(\varphi)\left(Q_{n} \varphi-\varphi\right)=R\left(Q_{n} \varphi-\varphi\right) .
$$

By (3.8)

$$
\left\|\left(I-Q_{n}\right) R\left(Q_{n} \varphi-\varphi\right)\right\|_{\infty} \leq C_{3}\left\|\left(R\left(Q_{n} \varphi-\varphi\right)\right)^{(r)}\right\|_{\infty} h^{r}
$$

We have

$$
\left(R\left(Q_{n} \varphi-\varphi\right)\right)^{(r)}(s)=\int_{0}^{1}(1-\theta)\left(S_{\theta}\left(Q_{n} \varphi-\varphi\right)\right)^{(r)}(s) d \theta
$$

where from (4.3),

$$
\begin{aligned}
& \left(S_{\theta}\left(Q_{n} \varphi-\varphi\right)\right)^{(r)}(s) \\
& \quad=\tilde{h} \sum_{j=1}^{m} \sum_{i=1}^{\rho} w_{i} \frac{\partial^{r+2} \kappa}{\partial s^{r} \partial u^{2}}\left(s, \zeta_{i}^{j}, \varphi\left(\zeta_{i}^{j}\right)+\theta\left(Q_{n} \varphi-\varphi\right)\left(\zeta_{i}^{j}\right)\right)\left(Q_{n} \varphi-\varphi\right)^{2}\left(\zeta_{i}^{j}\right)
\end{aligned}
$$

Let

$$
C_{8}=\max _{\substack{s, t \in[a, b] \\|u| \leq\|\varphi\|_{\infty}+\delta_{0}}}\left|\frac{\partial^{r+2} \kappa}{\partial s^{r} \partial u^{2}}(s, t, u)\right|
$$

Then

$$
\left\|\left(S_{\theta}\left(Q_{n} \varphi-\varphi\right)\right)^{(r)}\right\|_{\infty} \leq C_{8}(b-a)\left(\sum_{i=1}^{\rho}\left|w_{i}\right|\right)\left\|Q_{n} \varphi-\varphi\right\|_{\infty}^{2}
$$

and

$$
\left\|\left(R\left(Q_{n} \varphi-\varphi\right)\right)^{(r)}\right\|_{\infty} \leq \frac{C_{8}(b-a)}{2}\left(\sum_{i=1}^{\rho}\left|w_{i}\right|\right)\left\|Q_{n} \varphi-\varphi\right\|_{\infty}^{2} .
$$

Since $\kappa \in C^{r}(\Omega)$ and $f \in C^{r}[a, b]$, it follows that that $\varphi \in C^{r}[a, b]$. Hence by (3.8),

$$
\left\|Q_{n} \varphi-\varphi\right\|_{\infty} \leq C_{3}\left\|\varphi^{(r)}\right\|_{\infty} h^{r}
$$


Thus,

$$
\left\|\left(I-Q_{n}\right) R\left(Q_{n} \varphi-\varphi\right)\right\|_{\infty} \leq \frac{\left(C_{3}\right)^{3} C_{8}(b-a)}{2}\left(\sum_{i=1}^{\rho}\left|w_{i}\right|\right)\left\|\varphi^{(r)}\right\|_{\infty}^{2} h^{3 r},
$$

which completes the proof of (4.13).

In the following theorem we obtain the order of convergence in the discrete modified projection method.

Theorem 4.7. Let $r \geq 1, \kappa \in C^{d}(\Omega), \frac{\partial \kappa}{\partial u} \in C^{2 r}(\Omega)$ and $f \in C^{d}[a, b]$. Let $\varphi$ be the unique solution of (2.1) and assume that 1 is not an eigenvalue of $\mathscr{K}^{\prime}(\varphi)$. Let $n \geq n_{1}$ and $m \geq m_{1}$. Let $\mathscr{X}_{n}$ be the space of piecewise polynomials of degree $\leq r-1$ with respect to the partition (2.13) and $Q_{n}: L^{\infty}[0,1] \rightarrow \mathscr{X}_{n}$ be the interpolatory projection at $r$ Gauss points defined by (2.16)). Let $z_{n}^{M}$ be the unique solution of (2.21) in $B\left(\varphi, \delta_{0}\right)$. Then

$$
\left\|z_{n}^{M}-\varphi\right\|_{\infty}=O\left(\max \left\{\tilde{h}^{d}, h^{3 r}\right\}\right)
$$

Proof. From (4.12),

$$
\left\|z_{n}^{M}-\varphi\right\|_{\infty} \leq 3 C_{7}\left(\left\|\mathscr{K}(\varphi)-\mathscr{K}_{m}(\varphi)\right\|_{\infty}+\left\|\mathscr{K}_{m}(\varphi)-\tilde{\mathscr{K}}_{n}^{M}(\varphi)\right\|_{\infty}\right) .
$$

From (2.9),

$$
\left\|\mathscr{K}(\varphi)-\mathscr{K}_{m}(\varphi)\right\|_{\infty}=O\left(\tilde{h}^{d}\right) .
$$

Note that

$$
\begin{aligned}
\mathscr{K}_{m}(\varphi)-\tilde{\mathscr{K}}_{n}^{M}(\varphi)= & \left(I-Q_{n}\right)\left(\mathscr{K}_{m}(\varphi)-\mathscr{K}_{m}\left(Q_{n} \varphi\right)\right) \\
= & -\left(I-Q_{n}\right)\left(\mathscr{K}_{m}\left(Q_{n} \varphi\right)-\mathscr{K}_{m}(\varphi)-\mathscr{K}_{m}^{\prime}(\varphi)\left(Q_{n} \varphi-\varphi\right)\right) \\
& -\left(I-Q_{n}\right) \mathscr{K}_{m}^{\prime}(\varphi)\left(Q_{n} \varphi-\varphi\right) .
\end{aligned}
$$

Hence

$$
\begin{aligned}
\left\|\mathscr{K}_{m}(\varphi)-\tilde{\mathscr{K}}_{n}^{M}(\varphi)\right\|_{\infty} \leq & \left\|\left(I-Q_{n}\right)\left(\mathscr{K}_{m}\left(Q_{n} \varphi\right)-\mathscr{K}_{m}(\varphi)-\mathscr{K}_{m}^{\prime}(\varphi)\left(Q_{n} \varphi-\varphi\right)\right)\right\|_{\infty} \\
& +\left\|\left(I-Q_{n}\right) \mathscr{K}_{m}^{\prime}(\varphi)\left(Q_{n} \varphi-\varphi\right)\right\|_{\infty} .
\end{aligned}
$$


By (4.13) of Proposition 4.6,

$$
\left\|\left(I-Q_{n}\right)\left(\mathscr{K}_{m}\left(Q_{n} \varphi\right)-\mathscr{K}_{m}(\varphi)-\mathscr{K}_{m}^{\prime}(\varphi)\left(Q_{n} \varphi-\varphi\right)\right)\right\|_{\infty}=O\left(h^{3 r}\right)
$$

Define

$$
\ell(s, t)=\frac{\partial \kappa}{\partial u}(s, t, \varphi(t))
$$

Then from (4.5),

$$
\mathscr{K}_{m}^{\prime}(\varphi) v(s)=\tilde{h} \sum_{j=1}^{m} \sum_{i=1}^{\rho} w_{i} \ell\left(s, \zeta_{i}^{j}\right) v\left(\zeta_{i}^{j}\right), \quad s \in[a, b] .
$$

By assumption, $\frac{\partial \kappa}{\partial u} \in C^{2 r}(\Omega)$. Since $f \in C^{2 r}[a, b]$, it follows that $\varphi \in C^{2 r}[a, b]$. Hence $\ell \in C^{2 r}([a, b] \times[a, b])$. Thus, Proposition 3.4 is applicable and we obtain

$$
\left\|\left(I-Q_{n}\right) \mathscr{K}_{m}^{\prime}(\varphi)\left(Q_{n} \varphi-\varphi\right)\right\|_{\infty}=O\left(h^{3 r}\right)
$$

From (4.17)-(4.19), it follows that

$$
\left\|\mathscr{K}_{m}(\varphi)-\tilde{\mathscr{K}}_{n}^{M}(\varphi)\right\|_{\infty}=O\left(h^{3 r}\right) .
$$

The required result follows from (4.15), (4.16) and (4.20).

\section{Discrete Iterated Modified Projection method for Urysohn Integral Equations}

Recall from Remark 4.5 that there exists $\delta_{0}>0$ and a positive integer $n_{1}$ such that for $n \geq n_{1}$ equation (2.21) has a unique solution $z_{n}^{M}$ in $B\left(\varphi, \delta_{0}\right)$.

The discrete iterated modified projection solution is defined as

$$
\tilde{z}_{n}^{M}=\mathscr{K}_{m}\left(z_{n}^{M}\right)+f
$$

In this section we show that $\tilde{z}_{n}^{M} \rightarrow \varphi$ as $n \rightarrow \infty$ and obtain its order of convergence.

Proposition 5.1. Let $r \geq 1, \kappa \in C^{d}(\Omega), \frac{\partial \kappa}{\partial u} \in C^{2 r}(\Omega)$ and $f \in C^{d}[a, b]$. Let $\varphi_{m}$ denote the Nyström approximation of the exact solution $\varphi$ of the Urysohn integral equation (1.1)-(1.2). Then for $n \geq n_{1}$ and $m=n p$,

$$
\tilde{z}_{n}^{M}-\varphi_{m}=\mathscr{K}_{m}^{\prime}\left(\varphi_{m}\right)\left(z_{n}^{M}-\varphi_{m}\right)+O\left(\max \left\{\tilde{h}^{d}, h^{3 r}\right\}^{2}\right) .
$$


Proof. Since

$$
\varphi_{m}-\mathscr{K}_{m}\left(\varphi_{m}\right)=f
$$

it follows that

$$
\tilde{z}_{n}^{M}-\varphi_{m}=\mathscr{K}_{m}\left(z_{n}^{M}\right)-\mathscr{K}_{m}\left(\varphi_{m}\right) .
$$

In Theorem 4.7 we proved that

$$
\left\|z_{n}^{M}-\varphi\right\|_{\infty}=O\left(\max \left\{\tilde{h}^{d}, h^{3 r}\right\}\right)
$$

By Proposition 4.1 with $\delta=\delta_{0}$, for $s \in[a, b]$,

$$
\mathscr{K}_{m}\left(z_{n}^{M}\right)(s)-\mathscr{K}_{m}\left(\varphi_{m}\right)(s)=\mathscr{K}_{m}^{\prime}\left(\varphi_{m}\right)\left(z_{n}^{M}-\varphi_{m}\right)(s)+R\left(z_{n}^{M}-\varphi_{m}\right)(s),
$$

where

$$
\begin{aligned}
\left\|R\left(z_{n}^{M}-\varphi_{m}\right)\right\|_{\infty} & \leq \frac{C_{6}(b-a)}{2}\left(\sum_{i=1}^{\rho}\left|w_{i}\right|\right)\left\|z_{n}^{M}-\varphi_{m}\right\|_{\infty}^{2} \\
& =O\left(\max \left\{\tilde{h}^{d}, h^{3 r}\right\}^{2}\right) .
\end{aligned}
$$

The required result follows from (5.3)-(5.5) .

In order to obtain an error estimate for the first term in (5.2) we need to define a new operator which has $z_{n}^{M}$ as a fixed point. For this purpose, we show that for all $m$ large enough, $I-\mathscr{K}_{m}^{\prime}\left(\varphi_{m}\right)$ are invertible and are uniformly bounded.

Proposition 5.2. Let $\frac{\partial^{2} \kappa}{\partial u^{2}} \in C(\Omega)$. There exists a positive integer $m_{2} \geq m_{1}$ such that for $m \geq m_{2}$

$$
\left\|\left(I-\mathscr{K}_{m}^{\prime}\left(\varphi_{m}\right)\right)^{-1}\right\| \leq 2 C_{7}
$$

Proof. Recall from (4.7) that for $m \geq m_{1}$,

$$
\left\|\left(I-\mathscr{K}_{m}^{\prime}(\varphi)\right)^{-1}\right\| \leq C_{7}
$$

By Proposition 4.3 with $\delta=\delta_{0}$,

$$
\left\|\mathscr{K}_{m}^{\prime}(\varphi)-\mathscr{K}_{m}^{\prime}\left(\varphi_{m}\right)\right\| \leq \gamma\left\|\varphi-\varphi_{m}\right\|_{\infty} \rightarrow 0 \text { as } m \rightarrow \infty
$$


Choose $m_{2} \geq m_{1}$ such that

$$
m \geq m_{2} \Rightarrow\left\|\varphi-\varphi_{m}\right\|_{\infty} \leq \frac{1}{2 C_{7} \gamma}
$$

Since

$$
I-\mathscr{K}_{m}^{\prime}\left(\varphi_{m}\right)=\left(I-\left(\mathscr{K}_{m}^{\prime}\left(\varphi_{m}\right)-\mathscr{K}_{m}^{\prime}(\varphi)\right)\left(I-\mathscr{K}_{m}^{\prime}(\varphi)\right)^{-1}\right)\left(I-\mathscr{K}_{m}^{\prime}(\varphi)\right)
$$

it follows that for $m \geq m_{2}$,

$$
\left(I-\mathscr{K}_{m}^{\prime}\left(\varphi_{m}\right)\right)^{-1} \text { exists }
$$

and that

$$
\left\|\left(I-\mathscr{K}_{m}^{\prime}\left(\varphi_{m}\right)\right)^{-1}\right\| \leq 2 C_{7}
$$

This completes the proof.

Remark 5.3. In (4.10) we defined an operator $B_{n}$ which has a unique fixed point $z_{n}^{M}$ in $B\left(\varphi, \delta_{0}\right)$. Now we define another operator $\tilde{B}_{n}$ which also has $z_{n}^{M}$ as a fixed point.

From (2.20) recall that

$$
\tilde{\mathscr{K}}_{n}^{M}(x)=Q_{n} \mathscr{K}_{m}(x)+\mathscr{K}_{m}\left(Q_{n} x\right)-Q_{n} \mathscr{K}_{m}\left(Q_{n} x\right)
$$

For $m \geq m_{2}$, define

$$
\tilde{B}_{n}(x)=\varphi_{m}-\left[I-\mathscr{K}_{m}^{\prime}\left(\varphi_{m}\right)\right]^{-1}\left\{\mathscr{K}_{m}\left(\varphi_{m}\right)-\mathscr{K}_{m}^{\prime}\left(\varphi_{m}\right) \varphi_{m}-\tilde{\mathscr{K}}_{n}^{M}(x)+\mathscr{K}_{m}^{\prime}\left(\varphi_{m}\right) x\right\} .
$$

Then

$$
\begin{aligned}
& \tilde{B}_{n}(x)=x \\
\Leftrightarrow & \left(I-\mathscr{K}_{m}^{\prime}\left(\varphi_{m}\right)\right) \varphi_{m}-\left\{\mathscr{K}_{m}\left(\varphi_{m}\right)-\mathscr{K}_{m}^{\prime}\left(\varphi_{m}\right) \varphi_{m}-\tilde{\mathscr{K}}_{n}^{M}(x)+\mathscr{K}_{m}^{\prime}\left(\varphi_{m}\right) x\right\} \\
= & \left(I-\mathscr{K}_{m}^{\prime}\left(\varphi_{m}\right)\right) x \\
\Leftrightarrow & x-\tilde{\mathscr{K}}_{n}^{M}(x)=\varphi_{m}-\mathscr{K}_{m}\left(\varphi_{m}\right)=f .
\end{aligned}
$$

Thus, $x$ is a fixed point of $\tilde{B}_{n}$ if and only if it satisfies the equation (2.21).

Note that for $n \geq n_{1}$ and $m \geq m_{2}$,

$$
\begin{aligned}
z_{n}^{M}-\varphi_{m} & =\tilde{B}_{n}\left(z_{n}^{M}\right)-\varphi_{m} \\
& =-\left[I-\mathscr{K}_{m}^{\prime}\left(\varphi_{m}\right)\right]^{-1}\left\{\mathscr{K}_{m}\left(\varphi_{m}\right)-\mathscr{K}_{m}^{\prime}\left(\varphi_{m}\right) \varphi_{m}-\tilde{\mathscr{K}}_{n}^{M}\left(z_{n}^{M}\right)+\mathscr{K}_{m}^{\prime}\left(\varphi_{m}\right) z_{n}^{M}\right\} .
\end{aligned}
$$


Since $\mathscr{K}_{m}^{\prime}\left(\varphi_{m}\right)$ and $\left[I-\mathscr{K}_{m}^{\prime}\left(\varphi_{m}\right)\right]^{-1}$ commute, the first term in (5.2) can be written as

$$
\begin{aligned}
& \mathscr{K}_{m}^{\prime}\left(\varphi_{m}\right)\left(z_{n}^{M}-\varphi_{m}\right) \\
= & -\left[I-\mathscr{K}_{m}^{\prime}\left(\varphi_{m}\right)\right]^{-1} \mathscr{K}_{m}^{\prime}\left(\varphi_{m}\right)\left\{\mathscr{K}_{m}\left(\varphi_{m}\right)-\mathscr{K}_{m}^{\prime}\left(\varphi_{m}\right) \varphi_{m}-\tilde{\mathscr{K}}_{n}^{M}\left(z_{n}^{M}\right)+\mathscr{K}_{m}^{\prime}\left(\varphi_{m}\right) z_{n}^{M}\right\} .
\end{aligned}
$$

We write

$$
\begin{aligned}
& \mathscr{K}_{m}^{\prime}\left(\varphi_{m}\right)\left(z_{n}^{M}-\varphi_{m}\right) \\
= & -\left[I-\mathscr{K}_{m}^{\prime}\left(\varphi_{m}\right)\right]^{-1} \mathscr{K}_{m}^{\prime}\left(\varphi_{m}\right)\left\{\mathscr{K}_{m}\left(\varphi_{m}\right)-\tilde{\mathscr{K}}_{n}^{M}\left(\varphi_{m}\right)\right\} \\
& +\left[I-\mathscr{K}_{m}^{\prime}\left(\varphi_{m}\right)\right]^{-1} \mathscr{K}_{m}^{\prime}\left(\varphi_{m}\right)\left\{\tilde{\mathscr{K}}_{n}^{M}\left(z_{n}^{M}\right)-\tilde{\mathscr{K}}_{n}^{M}\left(\varphi_{m}\right)-\left(\tilde{\mathscr{K}}_{n}^{M}\right)^{\prime}\left(\varphi_{m}\right)\left(z_{n}^{M}-\varphi_{m}\right)\right\} \\
& +\left[I-\mathscr{K}_{m}^{\prime}\left(\varphi_{m}\right)\right]^{-1} \mathscr{K}_{m}^{\prime}\left(\varphi_{m}\right)\left\{\left(\left(\tilde{\mathscr{K}}_{n}^{M}\right)^{\prime}\left(\varphi_{m}\right)-\mathscr{K}_{m}^{\prime}\left(\varphi_{m}\right)\right)\left(z_{n}^{M}-\varphi_{m}\right)\right\} .
\end{aligned}
$$

We now obtain error estimates for the quantities appearing in the above expression.

Proposition 5.4. If $\frac{\partial \kappa}{\partial u} \in C^{3 r}(\Omega)$ and $x \in C^{2 r}[a, b]$, then for $n \geq n_{1}$ and $m \geq m_{2}$,

$$
\left\|\mathscr{K}_{m}^{\prime}\left(\varphi_{m}\right)\left(I-Q_{n}\right) \mathscr{K}_{m}^{\prime}\left(\varphi_{m}\right)\left(I-Q_{n}\right) x\right\|_{\infty}=O\left(h^{4 r}\right) .
$$

Proof. We have

$$
\mathscr{K}_{m}^{\prime}\left(\varphi_{m}\right) v(s)=\tilde{h} \sum_{j=1}^{m} \sum_{i=1}^{\rho} w_{i} \frac{\partial \kappa}{\partial u}\left(s, \zeta_{i}^{j}, \varphi_{m}\left(\zeta_{i}^{j}\right)\right) v\left(\zeta_{i}^{j}\right), \quad s \in[a, b]
$$

Let

$$
\ell_{m}(s, t)=\frac{\partial \kappa}{\partial u}\left(s, t, \varphi_{m}(t)\right), \quad s, t \in[a, b]
$$

Then $\ell_{m} \in C^{3 r}([a, b] \times[a, b])$ and Proposition 3.2 is applicable. Hence

$$
\left\|\mathscr{K}_{m}^{\prime}\left(\varphi_{m}\right)\left(I-Q_{n}\right) x\right\|_{\infty} \leq C_{4}\left\|\ell_{m}\right\|_{r, \infty}\|x\|_{2 r, \infty} h^{2 r}
$$

Let

$$
C_{9}=\max _{0 \leq i+j \leq r} \max _{\substack{s, t \in[a, b] \\|u| \leq\|\varphi\|_{\infty}+\delta_{0}}}\left|\frac{\partial^{i+j+1} \kappa}{\partial s^{i} \partial t^{j} \partial u}(s, t, u)\right| .
$$

Since for $m \geq m_{2}, \varphi_{m} \in B\left(\varphi, \delta_{0}\right)$, it follows that

$$
\left\|\ell_{m}\right\|_{r, \infty} \leq C_{9} .
$$


It then follows that

$$
\left\|\mathscr{K}_{m}^{\prime}\left(\varphi_{m}\right)\left(I-Q_{n}\right) x\right\|_{\infty} \leq C_{4} C_{9}\|x\|_{2 r, \infty} h^{2 r}
$$

Using the above estimate, we obtain

$$
\left\|\mathscr{K}_{m}^{\prime}\left(\varphi_{m}\right)\left(I-Q_{n}\right) \mathscr{K}_{m}^{\prime}\left(\varphi_{m}\right)\left(I-Q_{n}\right) x\right\|_{\infty} \leq C_{4} C_{9}\left\|\mathscr{K}_{m}^{\prime}\left(\varphi_{m}\right)\left(I-Q_{n}\right) x\right\|_{2 r, \infty} h^{2 r}
$$

For $0 \leq \beta \leq 2 r$,

$$
\left(\mathscr{K}_{m}^{\prime}\left(\varphi_{m}\right) v\right)^{(\beta)}(s)=\tilde{h} \sum_{j=1}^{m} \sum_{i=1}^{\rho} w_{i} \frac{\partial^{\beta+1} \kappa}{\partial s^{\beta} \partial u}\left(s, \zeta_{i}^{j}, \varphi_{m}\left(\zeta_{i}^{j}\right)\right) v\left(\zeta_{i}^{j}\right), \quad s \in[a, b] .
$$

Note that for $0 \leq \beta \leq 2 r$,

$$
\frac{\partial^{\beta+1} \kappa}{\partial s^{\beta} \partial u}\left(s, t, \varphi_{m}(t)\right)=D^{(\beta, 0)} \ell_{m}(s, t) \in C^{3 r-\beta}([a, b] \times[a, b]) .
$$

Let

$$
C_{10}=\max _{0 \leq i+j \leq 3 r} \max _{\substack{s, t \in[a, b] \\|u| \leq\|\varphi\|_{\infty}+\delta_{0}}}\left|\frac{\partial^{i+j+1} \kappa}{\partial s^{i} \partial t^{j} \partial u}(s, t, u)\right| .
$$

Then for $0 \leq \beta \leq 2 r$,

$$
\left\|D^{(\beta, 0)} \ell_{m}\right\|_{r, \infty} \leq C_{10}
$$

By Proposition $[3.2$, for $0 \leq \beta \leq 2 r$, we obtain

$$
\begin{aligned}
\left\|\left(\mathscr{K}_{m}^{\prime}\left(\varphi_{m}\right)\left(I-Q_{n}\right) x\right)^{(\beta)}\right\|_{\infty} & \leq C_{4}\left\|D^{(\beta, 0)} \ell_{m}\right\|_{r, \infty}\|x\|_{2 r, \infty} h^{2 r} \\
& \leq C_{4} C_{10}\|x\|_{2 r, \infty} h^{2 r} .
\end{aligned}
$$

Hence

$$
\begin{aligned}
\left\|\mathscr{K}_{m}^{\prime}\left(\varphi_{m}\right)\left(I-Q_{n}\right) x\right\|_{2 r, \infty} & =\max \left\{\left\|\left(\mathscr{K}_{m}^{\prime}\left(\varphi_{m}\right)\left(I-Q_{n}\right) x\right)^{(\beta)}\right\|_{\infty}: 0 \leq \beta \leq 2 r\right\} \\
& \leq C_{4} C_{10}\|x\|_{2 r, \infty} h^{2 r} .
\end{aligned}
$$

Thus, from (5.11) and (5.12) we obtain

$$
\left\|\mathscr{K}_{m}^{\prime}\left(\varphi_{m}\right)\left(I-Q_{n}\right) \mathscr{K}_{m}^{\prime}\left(\varphi_{m}\right)\left(I-Q_{n}\right) x\right\|_{\infty} \leq\left(C_{4}\right)^{2} C_{9} C_{10}\|x\|_{2 r, \infty} h^{4 r}
$$

which completes the proof. 
Proposition 5.5. If $\frac{\partial \kappa}{\partial u} \in C^{3 r}(\Omega)$, then for $n \geq n_{1}$ and $m \geq m_{2}$,

$$
\left\|\mathscr{K}_{m}^{\prime}\left(\varphi_{m}\right)\left(I-Q_{n}\right) \mathscr{K}_{m}^{\prime}\left(\varphi_{m}\right)\right\|=O\left(h^{2 r}\right)
$$

Proof. From (5.10)

$$
\left\|\mathscr{K}_{m}^{\prime}\left(\varphi_{m}\right)\left(I-Q_{n}\right) \mathscr{K}_{m}^{\prime}\left(\varphi_{m}\right) x\right\|_{2 r, \infty} \leq C_{4} C_{9}\left\|\mathscr{K}_{m}^{\prime}\left(\varphi_{m}\right) x\right\|_{2 r, \infty} h^{2 r} .
$$

For $0 \leq \beta \leq 2 r$,

$$
\left(\mathscr{K}_{m}^{\prime}\left(\varphi_{m}\right) x\right)^{(\beta)}(s)=\tilde{h} \sum_{j=1}^{m} \sum_{i=1}^{\rho} w_{i} \frac{\partial^{\beta+1} \kappa}{\partial s^{\beta} \partial u}\left(s, \zeta_{i}^{j}, \varphi_{m}\left(\zeta_{i}^{j}\right)\right) x\left(\zeta_{i}^{j}\right), \quad s \in[a, b] .
$$

Then

$$
\left\|\mathscr{K}_{m}^{\prime}\left(\varphi_{m}\right) x\right\|_{2 r, \infty} \leq(b-a) C_{10}\left(\sum_{i=1}^{\rho}\left|w_{i}\right|\right)\|x\|_{\infty}
$$

Thus,

$$
\left\|\mathscr{K}_{m}^{\prime}\left(\varphi_{m}\right)\left(I-Q_{n}\right) \mathscr{K}_{m}^{\prime}\left(\varphi_{m}\right) x\right\|_{\infty} \leq(b-a) C_{4} C_{9} C_{10}\left(\sum_{i=1}^{\rho}\left|w_{i}\right|\right)\|x\|_{\infty} h^{2 r}
$$

Taking the supremum over the set $\left\{x \in C[a, b]:\|x\|_{\infty} \leq 1\right\}$, we obtain

$$
\left\|\mathscr{K}_{m}^{\prime}\left(\varphi_{m}\right)\left(I-Q_{n}\right) \mathscr{K}_{m}^{\prime}\left(\varphi_{m}\right)\right\| \leq(b-a) C_{4} C_{9} C_{10}\left(\sum_{i=1}^{\rho}\left|w_{i}\right|\right) h^{2 r},
$$

which completes the proof.

Proposition 5.6. Let $r \geq 1, \kappa \in C^{d}(\Omega)$ and $f \in C^{d}[a, b]$. If $\frac{\partial^{2} \kappa}{\partial u^{2}} \in C^{2 r}(\Omega)$, then for $n \geq n_{1}$ and $m \geq m_{2}$

$$
\left\|\mathscr{K}_{m}^{\prime}\left(\varphi_{m}\right)\left(I-Q_{n}\right)\left[\mathscr{K}_{m}\left(Q_{n} \varphi_{m}\right)-\mathscr{K}_{m}\left(\varphi_{m}\right)-\mathscr{K}_{m}^{\prime}\left(\varphi_{m}\right)\left(Q_{n} \varphi_{m}-\varphi_{m}\right)\right]\right\|_{\infty}=O\left(h^{4 r}\right) .
$$

Proof. Let

$$
y_{n}=\mathscr{K}_{m}\left(Q_{n} \varphi_{m}\right)-\mathscr{K}_{m}\left(\varphi_{m}\right)-\mathscr{K}_{m}^{\prime}\left(\varphi_{m}\right)\left(Q_{n} \varphi_{m}-\varphi_{m}\right)=R\left(Q_{n} \varphi_{m}-\varphi_{m}\right) .
$$

By (5.10)

$$
\left\|\mathscr{K}_{m}^{\prime}\left(\varphi_{m}\right)\left(I-Q_{n}\right) y_{n}\right\|_{\infty} \leq C_{4} C_{9}\left\|y_{n}\right\|_{2 r, \infty} h^{2 r}
$$


Recall from (4.2) and (4.3) that

$$
R\left(v_{2}-v_{1}\right)(s)=\int_{0}^{1}(1-\theta) S_{\theta}\left(v_{2}-v_{1}\right)(s) d \theta,
$$

with

$$
S_{\theta}\left(v_{2}-v_{1}\right)(s)=\tilde{h} \sum_{j=1}^{m} \sum_{i=1}^{\rho} w_{i} \frac{\partial^{2} \kappa}{\partial u^{2}}\left(s, \zeta_{i}^{j}, v_{1}\left(\zeta_{i}^{j}\right)+\theta\left(v_{2}-v_{1}\right)\left(\zeta_{i}^{j}\right)\right)\left(v_{2}-v_{1}\right)^{2}\left(\zeta_{i}^{j}\right) .
$$

Let

$$
C_{11}=\max _{0 \leq \beta \leq 2 r} \max _{\substack{s, t \in[a, b] \\|u| \leq\|\varphi\|_{\infty}+\delta_{0}}}\left|\frac{\partial^{\beta+2} \kappa}{\partial s^{\beta} \partial u^{2}}(s, t, u)\right|
$$

Then for $0 \leq \beta \leq 2 r$,

$$
\left\|\left(R\left(v_{2}-v_{1}\right)\right)^{(\beta)}\right\|_{\infty} \leq \frac{C_{11}(b-a)}{2}\left(\sum_{i=1}^{\rho}\left|w_{i}\right|\right)\left\|v_{2}-v_{1}\right\|_{\infty}^{2}
$$

and

$$
\left\|y_{n}\right\|_{2 r, \infty}=\left\|R\left(Q_{n} \varphi_{m}-\varphi_{m}\right)\right\|_{2 r, \infty} \leq \frac{C_{11}(b-a)}{2}\left(\sum_{i=1}^{\rho}\left|w_{i}\right|\right)\left\|Q_{n} \varphi_{m}-\varphi_{m}\right\|_{\infty}^{2} .
$$

From (2.12), (2.17) and (3.8),

$$
\begin{aligned}
\left\|\left(Q_{n}-I\right) \varphi_{m}\right\|_{\infty} & \leq\left\|\left(Q_{n}-I\right) \varphi\right\|_{\infty}+\left\|\left(Q_{n}-I\right)\left(\varphi_{m}-\varphi\right)\right\|_{\infty} \\
& \leq C_{3}\left\|\varphi^{(r)}\right\|_{\infty} h^{r}+O\left(\tilde{h}^{d}\right) \\
& =O\left(h^{r}\right) .
\end{aligned}
$$

The required result then follows from (5.16) - (5.18).

Proposition 5.7. Let $\kappa \in C^{d}(\Omega), \frac{\partial \kappa}{\partial u} \in C^{2 r}(\Omega)$ and $f \in C^{d}[a, b]$. Then for $n \geq n_{1}$ and $m \geq m_{2}$,

$$
\left\|\tilde{\mathscr{K}}_{n}^{M}\left(z_{n}^{M}\right)-\tilde{\mathscr{K}}_{n}^{M}\left(\varphi_{m}\right)-\left(\tilde{\mathscr{K}}_{n}^{M}\right)^{\prime}\left(\varphi_{m}\right)\left(z_{n}^{M}-\varphi_{m}\right)\right\|_{\infty}=O\left(\max \left\{\tilde{h}^{d}, h^{3 r}\right\}^{2}\right) .
$$

Proof. Note that $\varphi_{m}, z_{n}^{M} \in B\left(\varphi_{m}, \delta_{0}\right)$. By the generalized Taylor's theorem,

$$
\begin{aligned}
\tilde{\mathscr{K}}_{n}^{M}\left(z_{n}^{M}\right)-\tilde{\mathscr{K}}_{n}^{M}\left(\varphi_{m}\right)-\left(\tilde{\mathscr{K}}_{n}^{M}\right)^{\prime}\left(\varphi_{m}\right)\left(z_{n}^{M}-\varphi_{m}\right) \\
\quad=\int_{0}^{1}(1-\theta)\left(\tilde{\mathscr{K}}_{n}^{M}\right)^{\prime \prime}\left(\varphi_{m}+\theta\left(z_{n}^{M}-\varphi_{m}\right)\right)\left(z_{n}^{M}-\varphi_{m}\right)^{2}(s) d \theta .
\end{aligned}
$$


Hence

$$
\begin{aligned}
& \left\|\tilde{\mathscr{K}}_{n}^{M}\left(z_{n}^{M}\right)-\tilde{\mathscr{K}}_{n}^{M}\left(\varphi_{m}\right)-\left(\tilde{\mathscr{K}}_{n}^{M}\right)^{\prime}\left(\varphi_{m}\right)\left(z_{n}^{M}-\varphi_{m}\right)\right\|_{\infty} \\
& \quad \leq \frac{1}{2} \max _{0 \leq \theta \leq 1}\left\|\left(\tilde{\mathscr{K}}_{n}^{M}\right)^{\prime \prime}\left(\varphi_{m}+\theta\left(z_{n}^{M}-\varphi_{m}\right)\right)\right\|\left\|z_{n}^{M}-\varphi_{m}\right\|_{\infty}^{2} .
\end{aligned}
$$

Note that for $x \in B\left(\varphi, \delta_{0}\right)$,

$$
\left(\tilde{\mathscr{K}}_{n}^{M}\right)^{\prime \prime}(x)=Q_{n} \mathscr{K}_{m}^{\prime \prime}(x)+\left(I-Q_{n}\right) \mathscr{K}_{m}^{\prime \prime}\left(Q_{n} x\right)\left(Q_{n} \otimes Q_{n}\right)
$$

where $Q_{n} \otimes Q_{n}: X \times X \rightarrow X \times X$ is defined as

$$
\left(Q_{n} \otimes Q_{n}\right)(v, w)=\left(Q_{n} v, Q_{n} w\right)
$$

From (2.8) for $s \in[a, b]$,

$$
\begin{aligned}
\left(\mathscr{K}_{m}\right)^{\prime \prime} & \left(\varphi_{m}+\theta\left(z_{n}^{M}-\varphi_{m}\right)\right)\left(v_{1}, v_{2}\right)(s) \\
& =\tilde{h} \sum_{j=1}^{m} \sum_{i=1}^{\rho} w_{i} \frac{\partial^{2} \kappa}{\partial u^{2}}\left(s, \zeta_{i}^{j},\left(\varphi_{m}+\theta\left(z_{n}^{M}-\varphi_{m}\right)\right)\left(\zeta_{i}^{j}\right)\right) v_{1}\left(\zeta_{i}^{j}\right) v_{2}\left(\zeta_{i}^{j}\right) .
\end{aligned}
$$

Then for $0 \leq \theta \leq 1$,

$$
\left\|\left(\mathscr{K}_{m}\right)^{\prime \prime}\left(\varphi_{m}+\theta\left(z_{n}^{M}-\varphi_{m}\right)\right)\right\| \leq C_{6}(b-a)\left(\sum_{i=1}^{\rho}\left|w_{i}\right|\right)
$$

where $C_{6}$ is defined in (4.1) with $\delta=\delta_{0}$.

In a similar manner, it can be shown that for $0 \leq \theta \leq 1$,

$$
\left\|\left(\mathscr{K}_{m}\right)^{\prime \prime}\left(Q_{n} \varphi_{m}+\theta\left(Q_{n} z_{n}^{M}-Q_{n} \varphi_{m}\right)\right)\right\| \leq C_{6}(b-a)\left(\sum_{i=1}^{\rho}\left|w_{i}\right|\right) .
$$

Hence

$$
\max _{0 \leq \theta \leq 1}\left\|\left(\tilde{\mathscr{K}}_{n}^{M}\right)^{\prime \prime}\left(\varphi_{m}+\theta\left(z_{n}^{M}-\varphi_{m}\right)\right)\right\| \leq C_{6}(b-a) q\left(1+q+q^{2}\right)\left(\sum_{i=1}^{\rho}\left|w_{i}\right|\right) .
$$

By Theorem 4.7 and by (2.12),

$$
\left\|z_{n}^{M}-\varphi_{m}\right\|_{\infty} \leq\left\|z_{n}^{M}-\varphi\right\|_{\infty}+\left\|\varphi-\varphi_{m}\right\|_{\infty}=O\left(\max \left\{\tilde{h}^{d}, h^{3 r}\right\}\right)
$$

The required result follows from (5.20), (5.21) and the above estimate.. 
Proposition 5.8. Let $\kappa \in C^{d}(\Omega), \frac{\partial \kappa}{\partial u} \in C^{3 r}(\Omega)$ and $f \in C^{d}[a, b]$. Then for $n \geq n_{1}$ and $m \geq m_{2}$,

$$
\left\|\mathscr{K}_{m}^{\prime}\left(\varphi_{m}\right)\left(\left(\tilde{\mathscr{K}}_{n}^{M}\right)^{\prime}\left(\varphi_{m}\right)-\mathscr{K}_{m}^{\prime}\left(\varphi_{m}\right)\right)\left(z_{n}^{M}-\varphi_{m}\right)\right\|_{\infty}=O\left(h^{r} \max \left\{\tilde{h}^{d}, h^{3 r}\right\}\right) .
$$

Proof. Note that

$$
\left(\tilde{\mathscr{K}}_{n}^{M}\right)^{\prime}\left(\varphi_{m}\right)=Q_{n} \mathscr{K}_{m}^{\prime}\left(\varphi_{m}\right)+\left(I-Q_{n}\right) \mathscr{K}_{m}^{\prime}\left(Q_{n} \varphi_{m}\right) Q_{n}
$$

Hence

$$
\begin{aligned}
\mathscr{K}_{m}^{\prime}\left(\varphi_{m}\right)\left(\left(\tilde{\mathscr{K}}_{n}^{M}\right)^{\prime}\left(\varphi_{m}\right)-\mathscr{K}_{m}^{\prime}\left(\varphi_{m}\right)\right) & =-\mathscr{K}_{m}^{\prime}\left(\varphi_{m}\right)\left(I-Q_{n}\right) \mathscr{K}_{m}^{\prime}\left(\varphi_{m}\right) \\
& +\mathscr{K}_{m}^{\prime}\left(\varphi_{m}\right)\left(I-Q_{n}\right) \mathscr{K}_{m}^{\prime}\left(Q_{n} \varphi_{m}\right) Q_{n} \\
& =\mathscr{K}_{m}^{\prime}\left(\varphi_{m}\right)\left(I-Q_{n}\right)\left(\mathscr{K}_{m}^{\prime}\left(Q_{n} \varphi_{m}\right)-\mathscr{K}_{m}^{\prime}\left(\varphi_{m}\right)\right) Q_{n} \\
& -\mathscr{K}_{m}^{\prime}\left(\varphi_{m}\right)\left(I-Q_{n}\right) \mathscr{K}_{m}^{\prime}\left(\varphi_{m}\right)\left(I-Q_{n}\right) .
\end{aligned}
$$

By Proposition 5.5 .

$$
\left\|\mathscr{K}_{m}^{\prime}\left(\varphi_{m}\right)\left(I-Q_{n}\right) \mathscr{K}_{m}^{\prime}\left(\varphi_{m}\right)\right\|=O\left(h^{2 r}\right)
$$

By (4.8) and (5.18),

$$
\left\|\mathscr{K}_{m}^{\prime}\left(Q_{n} \varphi_{m}\right)-\mathscr{K}_{m}^{\prime}\left(\varphi_{m}\right)\right\| \leq \gamma\left\|Q_{n} \varphi_{m}-\varphi_{m}\right\|_{\infty}=O\left(h^{r}\right) .
$$

Let

$$
C_{12}=\max _{\substack{s, t \in[a, b] \\|u| \leq\|\varphi\|_{\infty}+\delta_{0}}}\left|\frac{\partial \kappa}{\partial u}(s, t, u)\right|
$$

Then

$$
\left\|\mathscr{K}_{m}^{\prime}\left(\varphi_{m}\right) x\right\|_{\infty} \leq C_{12}(b-a)\left(\sum_{i=1}^{\rho}\left|w_{i}\right|\right)\|x\|_{\infty}
$$

Hence

$$
\left\|\mathscr{K}_{m}^{\prime}\left(\varphi_{m}\right)\right\| \leq C_{12}(b-a)\left(\sum_{i=1}^{\rho}\left|w_{i}\right|\right)
$$

Thus,

$$
\left\|\mathscr{K}_{m}^{\prime}\left(\varphi_{m}\right)\left(\left(\tilde{\mathscr{K}}_{n}^{M}\right)^{\prime}\left(\varphi_{m}\right)-\mathscr{K}_{m}^{\prime}\left(\varphi_{m}\right)\right)\right\|=O\left(h^{r}\right)
$$

Since

$$
\left\|z_{n}^{M}-\varphi\right\|_{\infty}=O\left(\max \left\{\tilde{h}^{d}, h^{3 r}\right\}\right)
$$

the required result follows. 
In the following theorem we obtain the order of convergence in the discrete iterated modified projection method.

Theorem 5.9. Let $r \geq 1, \kappa \in C^{d}(\Omega), \frac{\partial^{2} \kappa}{\partial u^{2}} \in C^{3 r}(\Omega)$ and $f \in C^{d}[a, b]$. Let $\varphi$ be the unique solution of (1.2) and assume that 1 is not an eigenvalue of $\mathscr{K}^{\prime}(\varphi)$. Let $\mathscr{X}_{n}$ be the space of piecewise polynomials of degree $\leq r-1$ with respect to the partition (2.13) and $Q_{n}: L^{\infty}[0,1] \rightarrow$ $\mathscr{X}_{n}$ be the interpolatory projection at $r$ Gauss points defined by (2.16). Let $z_{n}^{M}$ be the unique solution of (2.21) in $B\left(\varphi, \delta_{0}\right)$. Let $\tilde{z}_{n}^{M}$ be the discrete iterated modified projection solution defined by (5.1). Then

$$
\left\|\tilde{z}_{n}^{M}-\varphi\right\|_{\infty}=O\left(h^{r} \max \left\{\tilde{h}^{d}, h^{3 r}\right\}\right) .
$$

Proof. Recall from (5.2) that

$$
\tilde{z}_{n}^{M}-\varphi_{m}=\mathscr{K}_{m}^{\prime}\left(\varphi_{m}\right)\left(z_{n}^{M}-\varphi_{m}\right)+O\left(\max \left\{\tilde{h}^{d}, h^{3 r}\right\}^{2}\right)
$$

and from (5.8) that

$$
\begin{aligned}
& \mathscr{K}_{m}^{\prime}\left(\varphi_{m}\right)\left(z_{n}^{M}-\varphi_{m}\right) \\
= & -\left[I-\mathscr{K}_{m}^{\prime}\left(\varphi_{m}\right)\right]^{-1} \mathscr{K}_{m}^{\prime}\left(\varphi_{m}\right)\left\{\mathscr{K}_{m}\left(\varphi_{m}\right)-\tilde{\mathscr{K}}_{n}^{M}\left(\varphi_{m}\right)\right\} \\
& +\left[I-\mathscr{K}_{m}^{\prime}\left(\varphi_{m}\right)\right]^{-1} \mathscr{K}_{m}^{\prime}\left(\varphi_{m}\right)\left\{\tilde{\mathscr{K}}_{n}^{M}\left(z_{n}^{M}\right)-\tilde{\mathscr{K}}_{n}^{M}\left(\varphi_{m}\right)-\left(\tilde{\mathscr{K}}_{n}^{M}\right)^{\prime}\left(\varphi_{m}\right)\left(z_{n}^{M}-\varphi_{m}\right)\right\} \\
& +\left[I-\mathscr{K}_{m}^{\prime}\left(\varphi_{m}\right)\right]^{-1} \mathscr{K}_{m}^{\prime}\left(\varphi_{m}\right)\left\{\left(\left(\tilde{\mathscr{K}}_{n}^{M}\right)^{\prime}\left(\varphi_{m}\right)-\mathscr{K}_{m}^{\prime}\left(\varphi_{m}\right)\right)\left(z_{n}^{M}-\varphi_{m}\right)\right\} .
\end{aligned}
$$

From (5.6) we have

$$
\left\|\left[I-\mathscr{K}_{m}^{\prime}\left(\varphi_{m}\right)\right]^{-1}\right\| \leq 2 C_{7}
$$

Note that

$$
\begin{aligned}
\mathscr{K}_{m}\left(\varphi_{m}\right)-\tilde{\mathscr{K}}_{n}^{M}\left(\varphi_{m}\right)= & \left(I-Q_{n}\right)\left(\mathscr{K}_{m}\left(\varphi_{m}\right)-\mathscr{K}_{m}\left(Q_{n} \varphi_{m}\right)\right) \\
= & -\left(I-Q_{n}\right)\left(\mathscr{K}_{m}\left(Q_{n} \varphi_{m}\right)-\mathscr{K}_{m}\left(\varphi_{m}\right)-\mathscr{K}_{m}^{\prime}\left(\varphi_{m}\right)\left(Q_{n} \varphi_{m}-\varphi_{m}\right)\right) \\
& -\left(I-Q_{n}\right) \mathscr{K}_{m}^{\prime}\left(\varphi_{m}\right)\left(Q_{n} \varphi_{m}-\varphi_{m}\right) .
\end{aligned}
$$

By Proposition 5.6 .

$$
\| \mathscr{K}_{m}^{\prime}\left(\varphi_{m}\right)\left(I-Q_{n}\right)\left(\mathscr{K}_{m}\left(Q_{n} \varphi_{m}\right)-\mathscr{K}_{m}\left(\varphi_{m}\right)-\mathscr{K}_{m}^{\prime}\left(\varphi_{m}\right)\left(Q_{n} \varphi_{m}-\varphi_{m}\right) \|_{\infty}=O\left(h^{4 r}\right)\right.
$$


Since

$$
\begin{aligned}
\| \mathscr{K}_{m}^{\prime}\left(\varphi_{m}\right)(I- & \left.Q_{n}\right) \mathscr{K}_{m}^{\prime}\left(\varphi_{m}\right)\left(I-Q_{n}\right) \varphi_{m} \|_{\infty} \\
& \leq\left\|\mathscr{K}_{m}^{\prime}\left(\varphi_{m}\right)\left(I-Q_{n}\right) \mathscr{K}_{m}^{\prime}\left(\varphi_{m}\right)\left(I-Q_{n}\right) \varphi\right\|_{\infty} \\
& +(1+q)\left\|\mathscr{K}_{m}^{\prime}\left(\varphi_{m}\right)\left(I-Q_{n}\right) \mathscr{K}_{m}^{\prime}\left(\varphi_{m}\right)\right\|\left\|\varphi_{m}-\varphi\right\|_{\infty}
\end{aligned}
$$

by Proposition 5.4, Proposition 5.5 and the estimate (2.12), we obtain

$$
\left\|\mathscr{K}_{m}^{\prime}\left(\varphi_{m}\right)\left(I-Q_{n}\right) \mathscr{K}_{m}^{\prime}\left(\varphi_{m}\right)\left(I-Q_{n}\right) \varphi_{m}\right\|_{\infty}=O\left(h^{4 r}\right)
$$

It then follows that

$$
\left\|\left[I-\mathscr{K}_{m}^{\prime}\left(\varphi_{m}\right)\right]^{-1} \mathscr{K}_{m}^{\prime}\left(\varphi_{m}\right)\left(\mathscr{K}_{m}\left(\varphi_{m}\right)-\tilde{\mathscr{K}}_{n}^{M}\left(\varphi_{m}\right)\right)\right\|_{\infty}=O\left(h^{4 r}\right)
$$

By Proposition 5.7 .

$$
\left\|\tilde{\mathscr{K}}_{n}^{M}\left(z_{n}^{M}\right)-\tilde{\mathscr{K}}_{n}^{M}\left(\varphi_{m}\right)-\left(\tilde{\mathscr{K}}_{n}^{M}\right)^{\prime}\left(\varphi_{m}\right)\left(z_{n}^{M}-\varphi_{m}\right)\right\|_{\infty}=O\left(\max \left\{\tilde{h}^{d}, h^{3 r}\right\}^{2}\right) .
$$

whereas by Proposition 5.8 .

$$
\left\|\mathscr{K}_{m}^{\prime}\left(\varphi_{m}\right)\left\{\left(\left(\tilde{\mathscr{K}}_{n}^{M}\right)^{\prime}\left(\varphi_{m}\right)-\mathscr{K}_{m}^{\prime}\left(\varphi_{m}\right)\right)\left(z_{n}^{M}-\varphi_{m}\right)\right\}\right\|_{\infty}=O\left(h^{r} \max \left\{\tilde{h}^{d}, h^{3 r}\right\}\right) .
$$

The required result follows from (5.24)-(5.30).

\section{$6 \quad$ Numerical Results}

For the sake of illustration, we quote the following results from Grammont et al [11].

Consider

$$
\varphi(s)-\int_{0}^{1} \frac{d s}{s+t+\varphi(t)}=f(s), \quad 0 \leq s \leq 1,
$$

where $f$ is so chosen that

$$
\varphi(t)=\frac{1}{t+c}, \quad c>0
$$

is a solution of (6.1). 
We consider $X_{n}$ to be either the space of piecewise constant functions or piecewise linear functions with respect to the following uniform partition of $[0,1]$ :

$$
0<\frac{1}{n}<\frac{2}{n}<\cdots<\frac{n}{n}=1 .
$$

The projection $Q_{n}$ is chosen to be the interpolatory projection at $r$ Gauss points with $r=1$ or $r=2$. Hence by Theorem 4.7 and Theorem 5.9

$$
\left\|z_{n}^{M}-\varphi\right\|_{\infty}=O\left(\max \left\{\tilde{h}^{d}, h^{3 r}\right\}\right), \quad\left\|\tilde{z}_{n}^{M}-\varphi\right\|_{\infty}=O\left(h^{r} \max \left\{\tilde{h}^{d}, h^{3 r}\right\}\right) .
$$

If $X_{n}$ is the space of piecewise constant functions with respect to the partition (5.2), then we choose the composite Simpson rule with respect to the partition (6.2) to evaluate the integrals numerically. Then $\tilde{h}=h$ and $d=4$. Thus,

$$
\left\|z_{n}^{M}-\varphi\right\|_{\infty}=O\left(h^{3}\right), \quad\left\|\tilde{z}_{n}^{M}-\varphi\right\|_{\infty}=O\left(h^{4}\right) .
$$

If $X_{n}$ is the space of piecewise linear functions with respect to the partition (5.2), and the interpolation points are Gauss 2 points, then we choose the composite Gauss 2 point rule with respect to an uniform partition with $m=n^{2}$ subintervals as the approximate quadrature rule. Then, $\tilde{h}=h^{2}$ and $d=4$. Hence

$$
\left\|z_{n}^{M}-\varphi\right\|_{\infty}=O\left(h^{6}\right), \quad\left\|\tilde{z}_{n}^{M}-\varphi\right\|_{\infty}=O\left(h^{8}\right) .
$$

In the following tables $\delta_{m}$ and $\delta_{I M}$ denote the computed orders of convergence in the discrete modified projection method and the discrete iterated modified projection method, respectively.

Table 6.1

$$
\varphi(t)=\frac{1}{t+1}
$$

\begin{tabular}{|c|cc|cc|cc|cc|}
\hline & \multicolumn{4}{|c|}{ Piecewise Constant: $r=1$} & \multicolumn{4}{c|}{ Piecewise Linear: $r=2$} \\
\hline$n$ & $\left\|\varphi-z_{n}^{M}\right\|_{\infty}$ & $\delta_{M}$ & $\left\|\varphi-\tilde{z}_{n}^{M}\right\|_{\infty}$ & $\delta_{I M}$ & $\left\|\varphi-z_{n}^{M}\right\|_{\infty}$ & $\delta_{M}$ & $\left\|\varphi-\tilde{z}_{n}^{M}\right\|_{\infty}$ & $\delta_{I M}$ \\
\hline 2 & $8.46 \times 10^{-4}$ & & $2.38 \times 10^{-5}$ & & $5.06 \times 10^{-4}$ & & $6.47 \times 10^{-5}$ \\
4 & $1.03 \times 10^{-4}$ & 3.04 & $1.37 \times 10^{-6}$ & 4.12 & $1.07 \times 10^{-5}$ & 5.56 & $2.09 \times 10^{-7}$ & 8.27 \\
8 & $1.24 \times 10^{-5}$ & 3.05 & $8.18 \times 10^{-8}$ & 4.07 & $1.85 \times 10^{-7}$ & 5.86 & $8.45 \times 10^{-10}$ & 7.95 \\
16 & $1.45 \times 10^{-6}$ & 3.09 & $4.99 \times 10^{-9}$ & 4.04 & $3.07 \times 10^{-9}$ & 5.90 & $3.35 \times 10^{-12}$ & 7.98 \\
32 & $1.59 \times 10^{-7}$ & 3.19 & $3.08 \times 10^{-10}$ & 4.02 & $4.74 \times 10^{-11}$ & 6.02 & $1.34 \times 10^{-14}$ & 7.96 \\
\hline
\end{tabular}


Table 6.2: $\quad \varphi(t)=\frac{1}{t+0.1}$

\begin{tabular}{|c|cc|cc|cc|cc|}
\hline & \multicolumn{4}{|c|}{ Piecewise Constant: $r=1$} & \multicolumn{4}{c|}{ Piecewise Linear: $r=2$} \\
\hline$n$ & $\left\|\varphi-z_{n}^{M}\right\|_{\infty}$ & $\delta_{M}$ & $\left\|\varphi-\tilde{z}_{n}^{M}\right\|_{\infty}$ & $\delta_{I M}$ & $\left\|\varphi-z_{n}^{M}\right\|_{\infty}$ & $\delta_{M}$ & $\left\|\varphi-\tilde{z}_{n}^{M}\right\|_{\infty}$ & $\delta_{I M}$ \\
\hline 2 & $3.64 \times 10^{-4}$ & & $7.80 \times 10^{-6}$ & & $9.39 \times 10^{-5}$ & & $1.14 \times 10^{-4}$ \\
4 & $6.29 \times 10^{-5}$ & 2.53 & $4.20 \times 10^{-7}$ & 4.21 & $1.19 \times 10^{-4}$ & -0.35 & $2.84 \times 10^{-7}$ & 8.65 \\
8 & $6.85 \times 10^{-6}$ & 2.83 & $2.42 \times 10^{-8}$ & 4.12 & $3.30 \times 10^{-6}$ & 5.18 & $1.10 \times 10^{-9}$ & 8.01 \\
16 & $1.12 \times 10^{-6}$ & 2.99 & $1.45 \times 10^{-9}$ & 4.06 & $4.99 \times 10^{-8}$ & 6.05 & $4.35 \times 10^{-12}$ & 7.99 \\
32 & $1.27 \times 10^{-7}$ & 3.14 & $8.89 \times 10^{-11}$ & 4.03 & $7.00 \times 10^{-10}$ & 6.16 & $1.78 \times 10^{-14}$ & 7.93 \\
\hline
\end{tabular}

It is seen that the computed orders of convergence match well with the expected orders of convergence in (6.4) and (6.5).

\section{Conclusion}

In this paper we consider approximate solutions of Urysohn integral equations with smooth kernels using discrete versions of the Modified Projection Method and of the Iterated Modified Projection Method associated with an interpolatory projection at $r$ Gauss points. The interval $[a, b]$ is divided into $m$ subintervals each of length $\tilde{h}=\frac{b-a}{m}$ and a composite numerical quadrature with a degree of precision $d$ is chosen to replace all the integrals. The range of the interpolatory projection at $r$ Gauss points is a space of piecewise polynomials of degree $\leq r-1$ with respect to a uniform partition of $[a, b]$ with $n$ subintervals each of length $h=\frac{b-a}{n}$. The exact solution is denoted by $\varphi$ and the approximate solutions obtained by using the Discrete Modified Projection Method and the Discrete Iterated Modified Projection Method are denoted respectively by $z_{n}^{M}$ and $\tilde{z}_{n}^{M}$. We choose $m=p n$ where $p \in \mathbb{N}$. The following orders of convergence are proved:

$$
\left\|z_{n}^{M}-\varphi\right\|_{\infty}=O\left(\max \left\{\tilde{h}^{d}, h^{3 r}\right\}\right), \quad\left\|\tilde{z}_{n}^{M}-\varphi\right\|_{\infty}=O\left(h^{r} \max \left\{\tilde{h}^{d}, h^{3 r}\right\}\right) .
$$

Since the errors in the Modified Projection Method and the Iterated Modified Projection Method are respectively of the order of $h^{3 r}$ and $h^{4 r}$, these orders of convergence are preserved if the numerical quadrature formula is so chosen that $\tilde{h}^{d}=h^{3 r}$. Note that we have at our disposal $\tilde{h}$, that is $m$, and $d$ to achieve this equality. 
Acknowledgement: The first author would like to thank Indo-French Centre for Applied Mathematics (IFCAM) for the partial support.

\section{References}

[1] K. E. ATKINSON, The numerical evaluation of fixed points for completely continuous operators, SIAM J. of Numerical Analysis, 10 (1973), 799 - 807.

[2] K. E. ATKINSON, A Survey of Numerical Methods for Solving Nonlinear Integral Equations, J. Integral Eqns. Appl., 4 (1992), 15-46.

[3] K. E. ATKINSON, The Numerical Solutions of Integral Equations of the Second Kind, Cambridge University Press, Cambridge, U.K., 1997.

[4] K. E. ATKINSON and J. FLORES, The discrete collocation method for nonlinear integral equations, IMA J. of Numerical Analysis, 13 (1993), 195 - 213.

[5] K. ATKINSON, I. GRAHAM and I. SLOAN, Piecewise continuous collocation for Integral Equations, SIAM J. of Numerical Analysis, 20 (1983), pp. 172-186.

[6] K. E. ATKINSON and F. A. POTRA, Projection and iterated projection methods for nonlinear integral equations, SIAM J. Numer. Anal., 24 (1987), 1352 - 1373.

[7] Z. CHEN, G. LONG and N. GNANESHWAR, The discrete multi-projection method for Fredholm integral equations of the second kind, J. Integral Equations Appl. Volume (19), (2007), 143-162.

[8] S. D. CONTE and C. de BOOR, Elementary Numerical Analysis, An Algorithmic Approach, McGraw International Editions, 1981.

[9] L. GRAMMONT and R. P. KULKARNI, A superconvergent projection method for nonlinear compact operator equations, C. R. Acad. Sci. Paris, 342 (2006), 215-218.

[10] L. GRAMMONT, A Galerkin's perturbation type method to approximate a fixed point of a compact operator, International Journal of Pure and Applied Mathematics, 69 (2011), $1-14$. 
[11] L. GRAMMONT, R. P. KULKARNI and P. VASCONCELOS, Modified projection and the iterated modified projection methods for nonlinear integral equations, J. Integral Equations Appl. Volume 25, (2013), 481-516.

[12] M. A. KRASNOSElSkII, G. M. VAINIKKO, P. P. ZABREIKO, Ya. B. RUTITSKII and V. Ya.STETSENKO, Approximate Solution of Operator Equations, P. Noordhoff, Groningen, 1972.

[13] R. P. KULKARNI, A Superconvergence Result for Solutions of Compact Operator Equations, Bulletin of the Australian Mathematical Society, 68(2003), 517-528. 